\title{
Dark Energy and the Accelerating Universe
}

\author{
JoshuA A. Frieman \\ Center for Particle Astrophysics, Fermi National Accelerator Laboratory, P. O. \\ Box 500, Batavia, IL 60510; \\ Kavli Institute for Cosmological Physics, The University of Chicago, $5640 \mathrm{~S}$. \\ Ellis Ave., Chicago, IL 60637; email: frieman@fnal.gov
}

Michael S. TuRner

Kavli Institute for Cosmological Physics, The University of Chicago, 5640 S. Ellis Ave., Chicago, IL 60637; email: mturner@uchicago.edu

\author{
Dragan Huterer \\ Department of Physics, University of Michigan, 450 Church St., Ann Arbor, \\ MI, 48109; email: huterer@umich.edu
}
Key Words cosmology, cosmological constant, supernovae, galaxy clusters, large- scale structure, weak gravitational lensing

\begin{abstract}
The discovery ten years ago that the expansion of the Universe is accelerating put in place the last major building block of the present cosmological model, in which the Universe is composed of $4 \%$ baryons, $20 \%$ dark matter, and $76 \%$ dark energy. At the same time, it posed one of the most profound mysteries in all of science, with deep connections to both astrophysics and particle physics. Cosmic acceleration could arise from the repulsive gravity of dark energy - for example, the quantum energy of the vacuum - or it may signal that General Relativity breaks down on cosmological scales and must be replaced. We review the present observational evidence for cosmic acceleration and what it has revealed about dark energy, discuss the various theoretical ideas that have been proposed to explain acceleration, and describe the key observational probes that will shed light on this enigma in the coming years.
\end{abstract}

\section{CONTENTS}

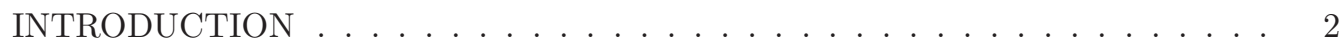

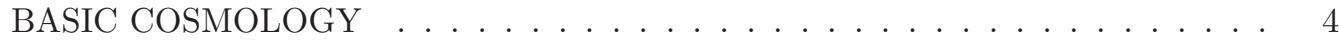

Friedmann-Robertson-Walker cosmology . . . . . . . . . . . . . 4

Distances and the Hubble diagram . . . . . . . . . . . . . . . . . . . . . . . . . . . . . .

Growth of structure and $\Lambda C D M \ldots \ldots \ldots \ldots$

FROM EINSTEIN TO ACCELERATED EXPANSION . . . . . . . . . . . 10

Greatest blunder? . . . . . . . . . . . . . . . . . . . . . . 10

Steady state and after . . . . . . . . . . . . . . . . . . . . . . . . . . . . . . . . . . . . . . . .

Enter inflation . . . . . . . . . . . . . . . . . . . . . 11 


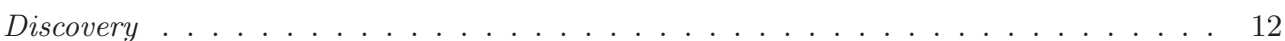

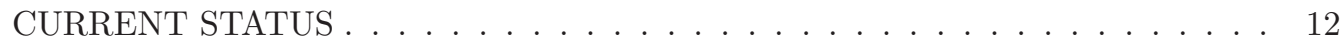

Cosmic microwave background and large-scale structure . . . . . . . . . . . 13

Recent supernova results . . . . . . . . . . . . . . . . . . . . . . 15

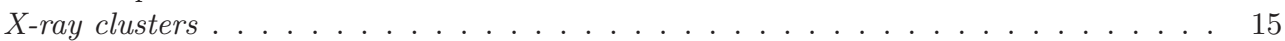

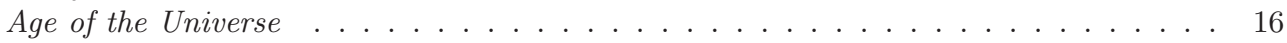

Cosmological parameters . . . . . . . . . . . . . . . . . . 17

UNDERSTANDING COSMIC ACCELERATION . . . . . . . . . . . . . 19

Dark energy models . . . . . . . . . . . . . . . . . . . . . . . . . . . 20

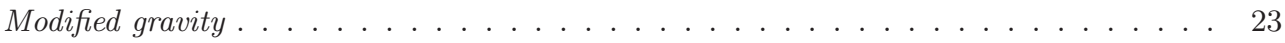

Unmodified gravity . . . . . . . . . . . . . . . . . . . . . . . . . . . . . . . . . . . . . . . . . . . .

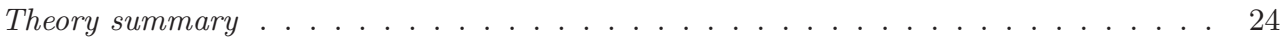

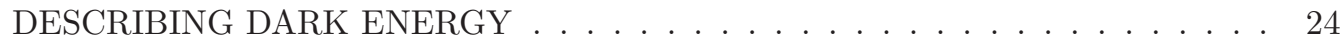

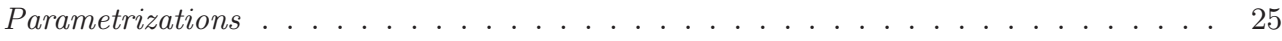

Direct reconstruction . . . . . . . . . . . . . . . . . . . 25

Principal components . . . . . . . . . . . . . . . . 26

Kinematic description . . . . . . . . . . . . . . . . . . . . 27

PROBES OF COSMIC ACCELERATION . . . . . . . . . . . . . . . . . . . . . . . . .

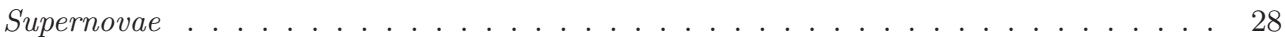

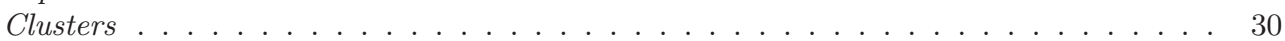

Baryon acoustic oscillations . . . . . . . . . . . . . . . . . . 32

Weak gravitational lensing . . . . . . . . . . . . . . . . . . 33

Other probes . . . . . . . . . . . . . . . . . . . . . . . 35

Role of the $C M B \ldots \ldots \ldots \ldots \ldots \ldots \ldots$

Probing new gravitational physics . . . . . . . . . . . . . . . . . . . . . . . . . . . . . . . . . . .

Summary and comparison ............................. 37

DARK ENERGY PROJECTS . . . . . . . . . . . . . 38

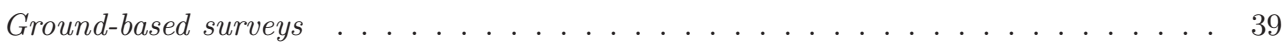

Space-based surveys . . . . . . . . . . . . . . . . . . . . . . . 40

DARK ENERGY \& COSMIC DESTINY . . . . . . . . . . . . . . . . . . 41

CONCLUDING REMARKS ...................... . . . 43

Take-home facts . . . . . . . . . . . . . . . . . . . . . . . . . . . . . . . . . . . . . . . .

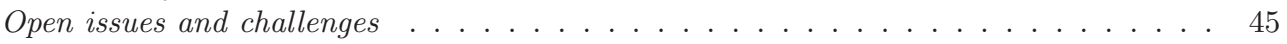

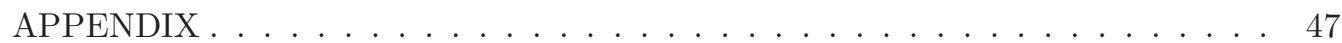

Figure $(s)$ of merit . . . . . . . . . . . . . . . . . . . . . . . . . . . . . . . .

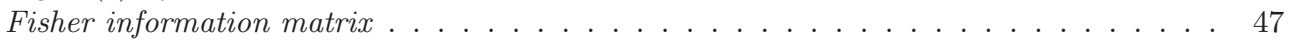

\section{INTRODUCTION}

In 1998, two teams studying distant Type Ia supernovae presented independent evidence that the expansion of the Universe is speeding up (Perlmutter et al. 1999, Riess et al. 1998). Since Hubble, cosmologists had been trying to measure the slowing of the expansion due to gravity; so expected was slow-down that the parameter used to quantify the second derivative of the expansion, $q_{0}$, was called the deceleration parameter (Sandage 1962). The discovery of cosmic acceleration is arguably one of the most important developments in modern cosmology.

The ready acceptance of the supernova results was not a foregone conclusion. 
The cosmological constant, the simplest explanation of accelerated expansion, had a checkered history, having been invoked and subsequently withdrawn several times before. This time, however, subsequent observations, including more detailed studies of supernovae and independent evidence from clusters of galaxies, large-scale structure, and the cosmic microwave background (CMB), confirmed and firmly established this remarkable finding.

The physical origin of cosmic acceleration remains a deep mystery. According to General Relativity (GR), if the Universe is filled with ordinary matter or radiation, the two known constituents of the Universe, gravity should lead to a slowing of the expansion. Since the expansion is speeding up, we are faced with two possibilities, either of which would have profound implications for our understanding of the cosmos and of the laws of physics. The first is that $75 \%$ of the energy density of the Universe exists in a new form with large negative pressure, called dark energy. The other possibility is that General Relativity breaks down on cosmological scales and must be replaced with a more complete theory of gravity.

Through a tangled history, dark energy is tied to Einstein's cosmological constant, $\Lambda$. Einstein introduced $\Lambda$ into the field equations of General Relativity in order to produce a static, finite cosmological model (Einstein 1917). With the discovery of the expansion of the Universe, the rationale for the cosmological constant evaporated. Fifty years later, Zel'dovich (1968) realized that $\Lambda$, mathematically equivalent to the stress-energy of empty space - the vacuum - cannot simply be dismissed. In quantum field theory, the vacuum state is filled with virtual particles, and their effects have been measured in the shifts of atomic lines and in particle masses. However, estimates for the energy density associated with the quantum vacuum are at least 60 orders of magnitude too large and in some cases infinite, a major embarrassment known as the cosmological constant problem (Weinberg 1989).

Despite the troubled history of $\Lambda$, the observational evidence for cosmic acceleration was quickly embraced by cosmologists, because it provided the missing element needed to complete the current cosmological model. In this model, the Universe is spatially flat and accelerating; composed of baryons, dark matter, and dark energy; underwent a hot, dense, early phase of expansion that produced the light elements via big bang nucleosynthesis and the CMB radiation; and experienced a much earlier epoch of accelerated expansion, known as inflation, which produced density perturbations from quantum fluctuations, leaving an imprint on the CMB anisotropy and leading by gravitational instability to the formation of large-scale structure.

The current cosmological model also raises deep issues, from the origin of the expansion itself and the nature of dark matter to the genesis of baryons and the cause of accelerated expansion. Of all these, the mystery of cosmic acceleration may be the richest, with broad connections to other important questions in cosmology and in particle physics. For example, the destiny of the Universe is tied to understanding dark energy; primordial inflation also involves accelerated expansion and its cause may be related; dark matter and dark energy could be linked; cosmic acceleration could provide a key to finding a successor to Einstein's theory of gravity; the smallness of the energy density of the quantum vacuum might reveal something about supersymmetry or even superstring theory; and the cause of cosmic acceleration could give rise to new long-range forces or be related to the smallness of neutrino masses. 
This review is organized into three parts. The first part is devoted to Context: in $\S 2$ we briefly review the Friedmann-Robertson-Walker (FRW) cosmology, the framework for understanding how observational probes of dark energy work. $\S 3$ provides the historical context, from Einstein's introduction of the cosmological constant to the supernova discovery. Part Two covers Current Status: in $\S 4$, we review the web of observational evidence that firmly establishes accelerated expansion. $\S 5$ summarizes current theoretical approaches to accelerated expansion and dark energy, including discussion of the cosmological constant problem, models of dark energy, and modified gravity, while $\S 6$ focuses on different phenomenological descriptions of dark energy and their relative merits. Part Three addresses The Future: $\S 7$ discusses the observational techniques that will be used to probe dark energy, primarily supernovae, weak lensing, large-scale structure, and clusters. In $\S 8$, we discuss specific projects aimed at constraining dark energy planned for the next fifteen years which have the potential to provide insights into the origin of cosmic acceleration. The connection between the future of the Universe and dark energy is the topic of $\S 9$. We summarize in $\S 10$, framing the two big questions about cosmic acceleration where progress should be made in the next fifteen years - Is dark energy something other than vacuum energy? Does General Relativity self-consistently describe cosmic acceleration? - and discussing what we believe are the most important open issues.

Our goal is to broadly review cosmic acceleration for the astronomy community. A number of useful reviews target different aspects of the subject, including: theory (Copeland. Sami \& Tsujikawa 2006; Padmanabhan 2003); cosmology (Peebles \& Ratra 2003); the physics of cosmic acceleration (Uzan 2007); probes of dark energy (Huterer \& Turner 2001): dark energy reconstruction (Sahni \& Starobinsky 2006); dynamics of dark energy models (Linder 2007); the cosmological constant (Carroll 2001; Carroll. Press \& Turner 1992), and the cosmological constant problem (Weinberg 1989).

\section{BASIC COSMOLOGY}

In this section, we provide a brief review of the elements of the FRW cosmological model. This model provides the context for interpreting the observational evidence for cosmic acceleration as well as the framework for understanding how cosmological probes in the future will help uncover the cause of acceleration by determining the history of the cosmic expansion with greater precision. For further details on basic cosmologv, see. e.g.. the textbooks of Dodelson (2003). Kolb \& Turner (1990), Peacock (1999), and Peebles (1993). Note that we follow the standard practice of using units in which the speed of light $c=1$.

\subsection{Friedmann-Robertson-Walker cosmology}

From the large-scale distribution of galaxies and the near-uniformity of the CMB temperature, we have good evidence that the Universe is nearly homogeneous and isotropic. Under this assumption, the spacetime metric can be written in the FRW form,

$$
d s^{2}=d t^{2}-a^{2}(t)\left[d r^{2} /\left(1-k r^{2}\right)+r^{2} d \theta^{2}+r^{2} \sin ^{2} \theta d \phi^{2}\right],
$$

where $r, \theta, \phi$ are comoving spatial coordinates, $t$ is time, and the expansion is described by the cosmic scale factor, $a(t)$ (by convention, $a=1$ today). The 
quantity $k$ is the curvature of 3 -dimensional space: $k=0$ corresponds to a spatially flat, Euclidean Universe, $k>0$ to positive curvature (3-sphere), and $k<0$ to negative curvature (saddle).

The wavelengths $\lambda$ of photons moving through the Universe scale with $a(t)$, and the redshift of light emitted from a distant source at time $t_{\mathrm{em}}, 1+z=$ $\lambda_{\text {obs }} / \lambda_{\mathrm{em}}=1 / a\left(t_{\mathrm{em}}\right)$, directly reveals the relative size of the Universe at that time. This means that time intervals are related to redshift intervals by $d t=$ $-d z / H(z)(1+z)$, where $H \equiv \dot{a} / a$ is the Hubble parameter, and an overdot denotes a time derivative. The present value of the Hubble parameter is conventionally expressed as $H_{0}=100 \mathrm{~h} \mathrm{~km} / \mathrm{sec} / \mathrm{Mpc}$, where $h \approx 0.7$ is the dimensionless Hubble parameter. Here and below, a subscript "0" on a parameter denotes its value at the present epoch.

The key equations of cosmology are the Friedmann equations, the field equations of GR applied to the FRW metric,

$$
\begin{aligned}
H^{2}=\left(\frac{\dot{a}}{a}\right)^{2} & =\frac{8 \pi G \rho}{3}-\frac{k}{a^{2}}+\frac{\Lambda}{3} \\
\frac{\ddot{a}}{a} & =-\frac{4 \pi G}{3}(\rho+3 p)+\frac{\Lambda}{3}
\end{aligned}
$$

where $\rho$ is the total energy density of the Universe (sum of matter, radiation, dark energy), and $p$ is the total pressure (sum of pressures of each component). For historical reasons we display the cosmological constant $\Lambda$ here; hereafter, we shall always represent it as vacuum energy and subsume it into the density and pressure terms; the correspondence is: $\Lambda=8 \pi G \rho_{\mathrm{VAC}}=-8 \pi G p_{\mathrm{VAC}}$.

For each component, the conservation of energy is expressed by $d\left(a^{3} \rho_{i}\right)=$ $-p_{i} d a^{3}$, the expanding Universe analogue of the first law of thermodynamics, $d E=-p d V$. Thus, the evolution of energy density is controlled by the ratio of the pressure to the energy density, the equation-of-state parameter, $w_{i} \equiv p_{i} / \rho_{i}$. 1 For the general case, this ratio varies with time, and the evolution of the energy density in a given component is given by

$$
\rho_{i} \propto \exp \left[3 \int_{0}^{z}\left[1+w_{i}\left(z^{\prime}\right)\right] d \ln \left(1+z^{\prime}\right)\right]
$$

In the case of constant $w_{i}$,

$$
w_{i} \equiv \frac{p_{i}}{\rho_{i}}=\text { constant }, \quad \rho_{i} \propto(1+z)^{3\left(1+w_{i}\right)} .
$$

For non-relativistic matter, which includes both dark matter and baryons, $w_{\mathrm{M}}=0$ to very good approximation, and $\rho_{\mathrm{M}} \propto(1+z)^{3}$; for radiation, i.e., relativistic particles, $w_{\mathrm{R}}=1 / 3$, and $\rho_{\mathrm{R}} \propto(1+z)^{4}$. For vacuum energy, as noted above $p_{\mathrm{VAC}}=-\rho_{\mathrm{VAC}}=-\Lambda / 8 \pi G=$ constant, i.e., $w_{\mathrm{VAC}}=-1$. For other models of

\footnotetext{
${ }^{1}$ A perfect fluid is fully characterized by its isotropic pressure $p$ and energy density $\rho$, where $p$ is a function of density and other state variables (e.g., temperature). The equation-of-state parameter $w=p / \rho$ determines the evolution of the energy density $\rho$; e.g., $\rho \propto V^{1+w}$ for constant $w$, where $V$ is the volume occupied by the fluid. Vacuum energy or a homogeneous scalar field are spatially uniform and they too can be fully characterized by $w$. The evolution of an inhomogeneous, imperfect fluid is in general complicated and not fully described by $w$. Nonetheless, in the FRW cosmology, spatial homogeneity and isotropy require the stress-energy to take the perfect fluid form; thus, $w$ determines the evolution of the energy density.
} 
dark energy, $w$ can differ from -1 and vary in time. [Hereafter, $w$ without a subscript refers to dark energy.]

The present energy density of a flat Universe $(k=0), \rho_{\text {crit }} \equiv 3 H_{0}^{2} / 8 \pi G=$ $1.88 \times 10^{-29} h^{2} \mathrm{gm} \mathrm{cm}{ }^{-3}=8.10 \times 10^{-47} h^{2} \mathrm{GeV}^{4}$, is known as the critical density; it provides a convenient means of normalizing cosmic energy densities, where $\Omega_{i}=\rho_{i}\left(t_{0}\right) / \rho_{\text {crit }}$. For a positively curved Universe, $\Omega_{0} \equiv \rho\left(t_{0}\right) / \rho_{\text {crit }}>1$ and for a negatively curved Universe $\Omega_{0}<1$. The present value of the curvature radius, $R_{\text {curv }} \equiv a / \sqrt{|k|}$, is related to $\Omega_{0}$ and $H_{0}$ by $R_{\text {curv }}=H_{0}^{-1} / \sqrt{\left|\Omega_{0}-1\right|}$, and the characteristic scale $H_{0}^{-1} \approx 3000 h^{-1} \mathrm{Mpc}$ is known as the Hubble radius. Because of the evidence from the CMB that the Universe is nearly spatially flat (see Fig. 8), we shall assume $k=0$ except where otherwise noted.

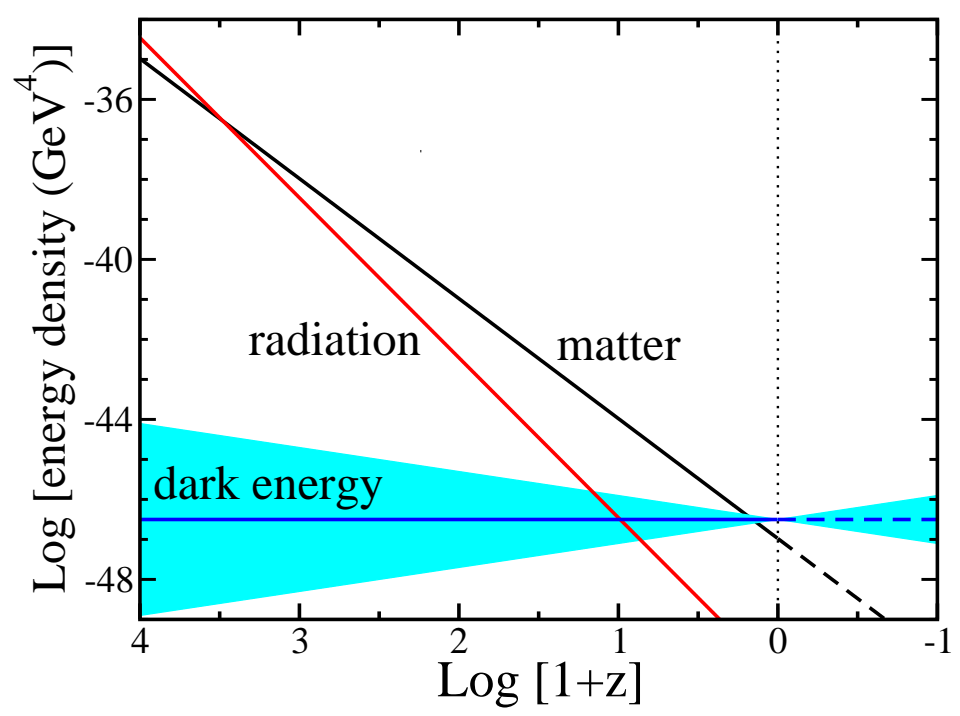

Figure 1: Evolution of radiation, matter, and dark energy densities with redshift. For dark energy, the band represents $w=-1 \pm 0.2$.

Fig. 1 shows the evolution of the radiation, matter, and dark energy densities with redshift. The Universe has gone through three distinct eras: radiationdominated, $z \gtrsim 3000$; matter-dominated, $3000 \gtrsim z \gtrsim 0.5$; and dark-energy dominated, $z \lesssim 0.5$. The evolution of the scale factor is controlled by the dominant energy form: $a(t) \propto t^{2 / 3(1+w)}$ (for constant $w$ ). During the radiationdominated era, $a(t) \propto t^{1 / 2}$; during the matter-dominated era, $a(t) \propto t^{2 / 3}$; and for the dark energy-dominated era, assuming $w=-1$, asymptotically $a(t) \propto$ $\exp (H t)$. For a flat Universe with matter and vacuum energy, the general solution, which approaches the latter two above at early and late times, is $a(t)=$ $\left(\Omega_{\mathrm{M}} / \Omega_{\mathrm{VAC}}\right)^{1 / 3}\left(\sinh \left[3 \sqrt{\Omega_{\mathrm{VAC}}} H_{0} t / 2\right]\right)^{2 / 3}$.

The deceleration parameter, $q(z)$, is defined as

$$
q(z) \equiv-\frac{\ddot{a}}{a H^{2}}=\frac{1}{2} \sum_{i} \Omega_{i}(z)\left[1+3 w_{i}(z)\right]
$$

where $\Omega_{i}(z) \equiv \rho_{i}(z) / \rho_{\text {crit }}(z)$ is the fraction of critical density in component $i$ at redshift $z$. During the matter- and radiation-dominated eras, $w_{i}>0$ and 
gravity slows the expansion, so that $q>0$ and $\ddot{a}<0$. Because of the $(\rho+$ $3 p)$ term in the second Friedmann equation (Newtonian cosmology would only have $\rho$ ), the gravity of a component that satisfies $p<-\rho / 3$, i.e., $w<-1 / 3$, is repulsive and can cause the expansion to accelerate $(\ddot{a}>0)$ : we take this to be the defining property of dark energy. The successful predictions of the radiationdominated era of cosmology, e.g., big bang nucleosynthesis and the formation of CMB anisotropies, provide evidence for the $(\rho+3 p)$ term, since during this epoch $\ddot{a}$ is about twice as large as it would be in Newtonian cosmology.

\subsection{Distances and the Hubble diagram}

For an object of intrinsic luminosity $L$, the measured energy flux $F$ defines the luminosity distance $d_{L}$ to the object, i.e., the distance inferred from the inverse square law. The luminosity distance is related to the cosmological model through

$$
d_{L}(z) \equiv \sqrt{\frac{L}{4 \pi F}}=(1+z) r(z)
$$

where $r(z)$ is the comoving distance to an object at redshift $z$,

$$
\begin{aligned}
& r(z)=\int_{0}^{z} \frac{d z^{\prime}}{H\left(z^{\prime}\right)}=\int_{1 /(1+z)}^{1} \frac{d a}{a^{2} H(a)} \quad(k=0), \\
& r(z)=|k|^{-1 / 2} \chi\left[|k|^{1 / 2} \int_{0}^{z} d z^{\prime} / H\left(z^{\prime}\right)\right] \quad(k \neq 0),
\end{aligned}
$$

and where $\chi(x)=\sin (x)$ for $k>0$ and $\sinh (x)$ for $k<0$. Specializing to the flat model and constant $w$,

$$
r(z)=\frac{1}{H_{0}} \int_{0}^{z} \frac{d z^{\prime}}{\sqrt{\Omega_{\mathrm{M}}\left(1+z^{\prime}\right)^{3}+\left(1-\Omega_{\mathrm{M}}\right)\left(1+z^{\prime}\right)^{3(1+w)}+\Omega_{\mathrm{R}}\left(1+z^{\prime}\right)^{4}}}
$$

where $\Omega_{\mathrm{M}}$ is the present fraction of critical density in non-relativistic matter, and $\Omega_{\mathrm{R}} \simeq 0.8 \times 10^{-4}$ represents the small contribution to the present energy density from photons and relativistic neutrinos. In this model, the dependence of cosmic distances upon dark energy is controlled by the parameters $\Omega_{\mathrm{M}}$ and $w$ and is shown in the left panel of Fig. 2 .

The luminosity distance is related to the distance modulus $\mu$ by

$$
\mu(z) \equiv m-M=5 \log _{10}\left(d_{L} / 10 \mathrm{pc}\right)=5 \log _{10}[(1+z) r(z) / \mathrm{pc}]-5,
$$

where $m$ is the apparent magnitude of the object (proportional to the log of the flux) and $M$ is the the absolute magnitude (proportional to the log of the intrinsic luminosity). "Standard candles," objects of fixed absolute magnitude $M$, and measurements of the logarithmic energy flux $m$ constrain the cosmological model and thereby the expansion history through this magnitude-redshift relation, known as the Hubble diagram.

Expanding the scale factor around its value today, $a(t)=1+H_{0}\left(t-t_{0}\right)-$ $q_{0} H_{0}^{2}\left(t-t_{0}\right)^{2} / 2+\cdots$, the distance-redshift relation can be written in its historical form

$$
H_{0} d_{L}=z+\frac{1}{2}\left(1-q_{0}\right) z^{2}+\cdots
$$

The expansion rate and deceleration rate today appear in the first two terms in the Taylor expansion of the relation. This expansion, only valid for $z \ll 1$, is of 

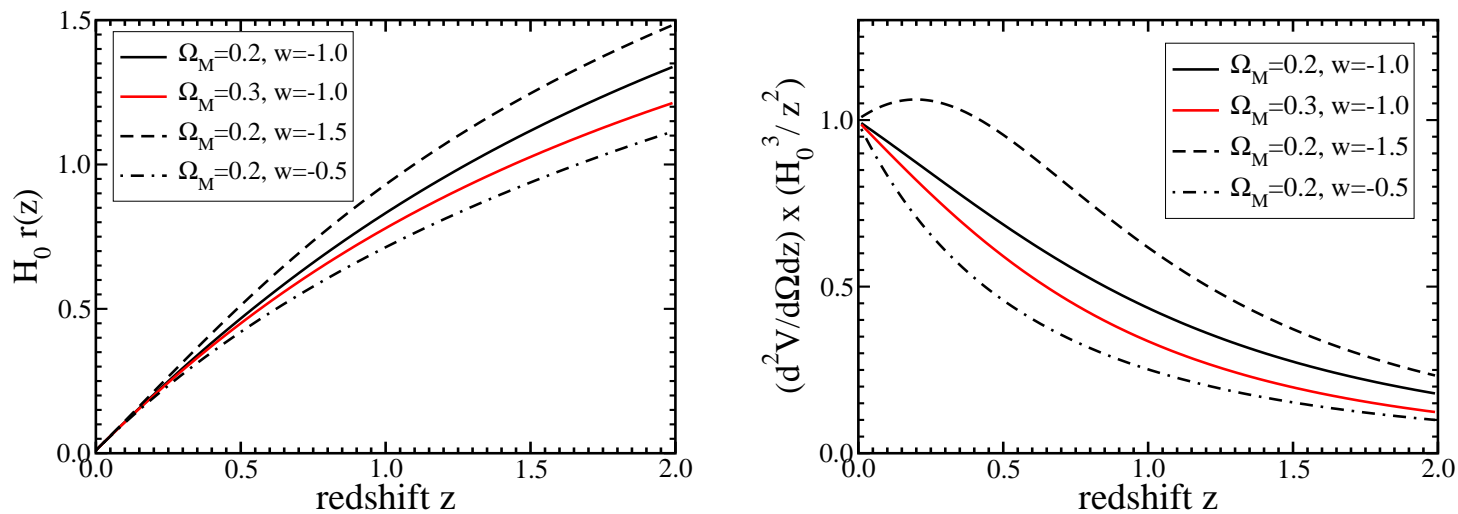

Figure 2: For a flat Universe, the effect of dark energy upon cosmic distance (left) and volume element (right) is controlled by $\Omega_{\mathrm{M}}$ and $w$.

historical significance and utility; it is not useful today since objects as distant as redshift $z \sim 2$ are being used to probe the expansion history. However, it does illustrate the general principle: the first term on the r.h.s. represents the linear Hubble expansion, and the deviation from a linear relation reveals the deceleration (or acceleration).

The angular-diameter distance $d_{A}$, the distance inferred from the angular size $\delta \theta$ of a distant object of fixed diameter $D$, is defined by $d_{A} \equiv D / \delta \theta=r(z) /(1+$ $z)=d_{L} /(1+z)^{2}$. The use of "standard rulers" (objects of fixed intrinsic size) provides another means of probing the expansion history, again through $r(z)$.

The cosmological time, or time back to the Big Bang, is given by

$$
t(z)=\int_{0}^{t(z)} d t^{\prime}=\int_{z}^{\infty} \frac{d z^{\prime}}{\left(1+z^{\prime}\right) H\left(z^{\prime}\right)}
$$

While the present age in principle depends upon the expansion rate at very early times, the rapid rise of $H(z)$ with $z-$ a factor of 30,000 between today and the epoch of last scattering, when photons and baryons decoupled, at $z_{L S} \simeq 1100$, $t\left(z_{L S}\right) \simeq 380,000$ years - makes this point moot.

Finally, the comoving volume element per unit solid angle $d \Omega$ is given by

$$
\frac{d^{2} V}{d z d \Omega}=r^{2} \frac{d r}{d z} \frac{1}{\sqrt{1-k r^{2}}}=\frac{r^{2}(z)}{H(z)} .
$$

For a set of objects of known comoving density $n(z)$, the comoving volume element can be used to infer $r^{2}(z) / H(z)$ from the number counts per unit redshift and solid angle, $d^{2} N / d z d \Omega=n(z) d^{2} V / d z d \Omega$. The dependence of the comoving volume element upon $\Omega_{\mathrm{M}}$ and $w$ is shown in the right panel of Fig. 2 ,

\subsection{Growth of structure and $\Lambda \mathrm{CDM}$}

A striking success of the consensus cosmology is its ability to account for the observed structure in the Universe, provided that the dark matter is composed of 


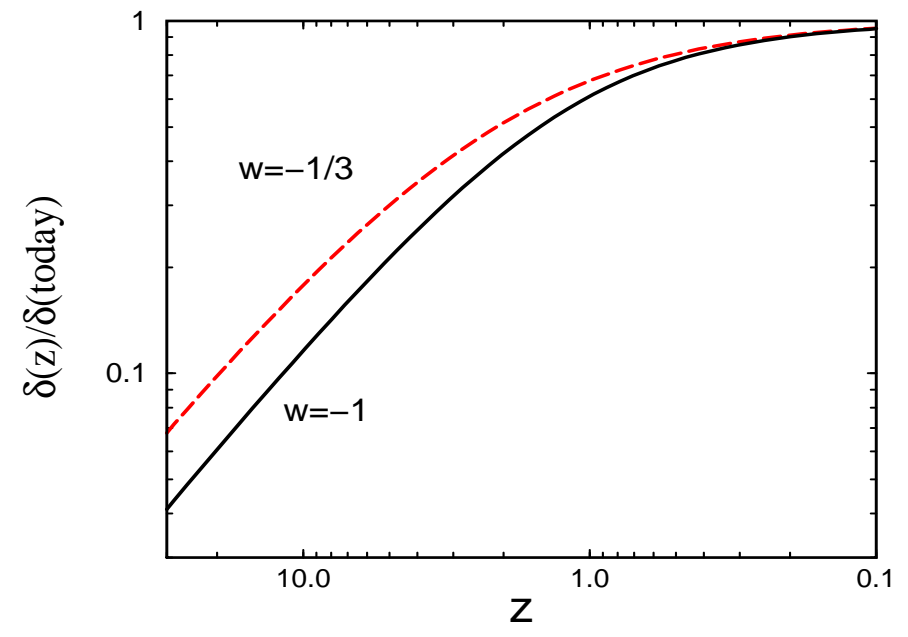

Figure 3: Growth of linear density perturbations in a flat universe with dark energy. Note that the growth of perturbations ceases when dark energy begins to dominate, $1+z=\left(\Omega_{\mathrm{M}} / \Omega_{\mathrm{DE}}\right)^{1 / 3 w}$.

slowly moving particles, known as cold dark matter (CDM), and that the initial power spectrum of density perturbations is nearly scale-invariant, $P(k) \sim k^{n_{S}}$ with spectral index $n_{S} \simeq 1$, as predicted by inflation (Springel. Frenk \& White 2006). Dark energy affects the development of structure by its influence on the expansion rate of the Universe when density perturbations are growing. This fact and the quantity and quality of large-scale structure data make structure formation a sensitive probe of dark energy.

In GR the growth of small-amplitude, matter-density perturbations on length scales much smaller than the Hubble radius is governed by

$$
\ddot{\delta}_{k}+2 H \dot{\delta}_{k}-4 \pi G \rho_{\mathrm{M}} \delta_{k}=0,
$$

where the perturbations $\delta(\mathbf{x}, t) \equiv \delta \rho_{\mathrm{M}}(\mathbf{x}, t) / \bar{\rho}_{\mathrm{M}}(t)$ have been decomposed into their Fourier modes of wavenumber $k$, and matter is assumed to be pressureless (always true for the CDM portion and valid for the baryons on mass scales larger than $10^{5} M_{\odot}$ after photon-baryon decoupling). Dark energy affects the growth through the "Hubble damping" term, $2 H \dot{\delta}_{k}$.

The solution to Eq. (15) is simple to describe during the three epochs of expansion discussed earlier: $\delta_{k}(t)$ grows as $a(t)$ during the matter-dominated epoch and is approximately constant during the radiation-dominated and dark energydominated epochs. The key feature here is the fact that once accelerated expansion begins, the growth of linear perturbations effectively ends, since the Hubble damping time becomes shorter than the timescale for perturbation growth.

The impact of the dark energy equation-of-state parameter $w$ on the growth of structure is more subtle and is illustrated in Fig. 3. For larger $w$ and fixed dark energy density $\Omega_{\mathrm{DE}}$, dark energy comes to dominate earlier, causing the growth of linear perturbations to end earlier; this means the growth factor since decoupling is smaller and that to achieve the same amplitude by today, the perturbation must begin with larger amplitude and is larger at all redshifts until today. The same is true for larger $\Omega_{\mathrm{DE}}$ and fixed $w$. Finally, if dark energy is dynamical (not vacuum energy), then in principle it can be inhomogeneous, an effect ignored 
above. In practice, it is expected to be nearly uniform over scales smaller than the present Hubble radius, in sharp contrast to dark matter, which can clump on small scales.

\section{FROM EINSTEIN TO ACCELERATED EXPANSION}

Although the discovery of cosmic acceleration is often portrayed as a major surprise and a radical contravention of the conventional wisdom, it was anticipated by a number of developments in cosmology in the preceding decade. Moreover, this is not the first time that the cosmological constant has been proposed. Indeed, the cosmological constant was explored from the very beginnings of General Relativity and has been periodically invoked and subsequently cast aside several times since. Here we recount some of this complex 90-year history.

\subsection{Greatest blunder?}

Einstein introduced the cosmological constant in his field equations in order to obtain a static and finite cosmological solution "as required by the fact of the small velocities of the stars" and to be consistent with Mach's principle (Einstein 1917). In Einstein's solution, space is positively curved, $R_{\text {curv }}=1 / \sqrt{4 \pi G \rho_{\mathrm{M}}}$, and the "repulsive gravity" of $\Lambda$ is balanced against the attractive gravity of matter, $\rho_{\Lambda}=\rho_{\mathrm{M}} / 2$. In the 1920's, Friedmann and Lemaitre independently showed that cosmological solutions with matter and $\Lambda$ generally involved expansion or contraction, and Lemaître as well as Eddington showed that Einstein's static solution was unstable to expansion or contraction. In 1917, de Sitter explored a solution in which $\rho_{\mathrm{M}}$ is negligible compared to $\rho_{\Lambda}$ (de Sitter 1917). There was some early confusion about the interpretation of this model, but in the early 1920's, Weyl, Eddington, and others showed that the apparent recession velocity (the redshift) at small separation would be proportional to the distance, $v=\sqrt{\Lambda / 3} d$.

With Hubble's discovery of the expansion of the Universe in 1929, Einstein's primary justification for introducing the cosmological constant was lost, and he advocated abandoning it. Gamow later wrote that Einstein called this "his greatest blunder," since he could have predicted the expanding Universe. Yet the description above makes it clear that the history was more complicated, and one could argue that in fact Friedmann and Lemaitre (or de Sitter) had "predicted" the expanding Universe, $\Lambda$ or no. Indeed, Hubble noted that his linear relation between redshift and distance was consistent with the prediction of the de Sitter model (Hubble 1929). Moreover, Eddington recognized that Hubble's value for the expansion rate, $H_{0} \simeq 570 \mathrm{~km} / \mathrm{s} / \mathrm{Mpc}$, implied a time back to the big bang of less than $2 \mathrm{Gyr}$, uncomfortably short compared to some age estimates of Earth and the galaxy. By adjusting the cosmological constant to be slightly larger than the Einstein value, $\rho_{\Lambda}=(1+\epsilon) \rho_{\mathrm{M}} / 2$, a nearly static beginning of arbitrary duration could be obtained, a solution known as the Eddington-Lemaitre model. While Eddington remained focused on $\Lambda$, trying to find a place for it in his "unified" and "fundamental" theories, $\Lambda$ was no longer the focus of most cosmologists. 


\subsection{Steady state and after}

Motivated by the aesthetic beauty of an unchanging Universe, Bondi \& Gold (1948) and Hoyle (1948) put forth the steady-state cosmology, a revival of the de Sitter model with a new twist. In the steady-state model, the dilution of matter due to expansion is counteracted by postulating the continuous creation of matter (about 1 hydrogen atom $/ \mathrm{m}^{3} / \mathrm{Gyr}$ ). However, the model's firm prediction of an unevolving Universe made it easily falsifiable, and the redshift distribution of radio galaxies, the absence of quasars nearby, and the discovery of the cosmic microwave background radiation did so in the early 1960s.

$\Lambda$ was briefly resurrected again in the late 1960 s by Petrosian, Salpeter \& Szekeres (1967), who used the Eddington-Lemaitre model to explain the preponderance of quasars at redshifts around $z \sim 2$. As it turns out, this is a real observational effect, but it can be attributed to evolution: quasar activity peaks around this redshift. In 1975, evidence for a cosmological constant from the Hubble diagram of brightest-cluster elliptical galaxies was presented (Gunn \& Tinsley 1975), though it was realized (Tinsley \& Gunn 1976) that uncertainties in galaxy luminosity evolution make their use as standard candles problematic.

While cosmologists periodically hauled the cosmological constant out of the closet as needed and then stuffed it back in, in the 1960s physicists began to understand that $\Lambda$ cannot be treated in such cavalier fashion. With the rise of the standard big-bang cosmology came the awareness that the cosmological constant could be a big problem (Zel'dovich 1968). It was realized that the energy density of the quantum vacuum should result in a cosmological constant of enormous size (see \$5.1.1). However, because of the success of the hot big-bang model, the lack of compelling ideas to solve the cosmological constant problem, and the dynamical unimportance of $\Lambda$ at the early epochs when the hot big-bang model was best tested by big-bang nucleosynthesis and the CMB, the problem was largely ignored in cosmological discourse.

\subsection{Enter inflation}

In the early 1980s the inflationary universe scenario (Guth 1981), with its predictions of a spatially flat Universe $(\Omega=1)$ and almost-scale-invariant density perturbations, changed the cosmological landscape and helped set the stage for the discovery of cosmic acceleration. When inflation was first introduced, the evidence for dark matter was still accruing, and estimates of the total matter density, then about $\Omega_{\mathrm{M}} \sim 0.1$, were sufficiently uncertain that an Einstein-de Sitter model (i.e., $\Omega_{\mathrm{M}}=1$ ) was not ruled out. The evidence for a low value of $\Omega_{\mathrm{M}}$ was, however, sufficiently worrisome that the need for a smooth component, such as vacuum energy, to make up the difference for a flat Universe was suggested (Peebles 1984; Turner. Steigman \& Krauss 1984). Later, the model for large-scale structure formation with a cosmological constant and cold dark matter $(\Lambda \mathrm{CDM})$ and the spectrum of density perturbations predicted by inflation was found to provide a better fit (than $\Omega_{\mathrm{M}}=1$ ) to the growing observations of large-scale structure (Efstathiou. Sutherland \& Maddox 1990; Turner 1991). The 1992 COBE discovery of CMB anisotropy provided the normalization of the spectrum of density perturbations and drove a spike into the heart of the $\Omega_{M}=1$ CDM model.

Another important thread involved age consistency. While estimates of the 
Hubble parameter had ranged between 50 and $100 \mathrm{~km} / \mathrm{s} / \mathrm{Mpc}$ since the 1970s, by the mid-1990s they were settling out in the middle of that range. Estimates of old globular cluster ages had similar swings, but had settled at $t_{0} \simeq 13-15$ Gyr. The resulting expansion age, $H_{0} t_{0}=\left(H_{0} / 70 \mathrm{~km} / \mathrm{s} / \mathrm{Mpc}\right)\left(t_{0} / 14 \mathrm{Gyr}\right)$ was uncomfortably high compared to that for the Einstein-de Sitter model, for which $H_{0} t_{0}=2 / 3$. The cosmological constant offered a ready solution, as the age of a flat Universe with $\Lambda$ rises with $\Omega_{\Lambda}$,

$$
H_{0} t_{0}=\frac{1}{3 \Omega_{\Lambda}^{1 / 2}} \ln \left[\frac{1+\Omega_{\Lambda}{ }^{1 / 2}}{1-\Omega_{\Lambda}{ }^{1 / 2}}\right]=\frac{2}{3}\left[1+\Omega_{\Lambda}{ }^{2} / 3+\Omega_{\Lambda}{ }^{4} / 5+\cdots\right],
$$

reaching $H_{0} t_{0} \simeq 1$ for $\Omega_{\Lambda}=0.75$.

By 1995 the cosmological constant was back out of the cosmologists' closet in full glory (Frieman et al. 1995, Krauss \& Turner 1995, Ostriker \& Steinhardt 1995): it solved the age problem, was consistent with growing evidence that $\Omega_{M}$ was around 0.3 , and fit the growing body of observations of large-scale structure. Its only serious competitors were "open inflation," which had a small group of adherents, and hot + cold dark matter, with a low value for the Hubble parameter $(\sim 50 \mathrm{~km} / \mathrm{s} / \mathrm{Mpc})$ and neutrinos accounting for $10 \%$ to $15 \%$ of the dark matter (see, e.g., contributions in Turok 1997). During this period, there were two results that conflicted with $\Lambda \mathrm{CDM}$ : analysis of the statistics of lensed quasars (Kochanek 1996) and of the first 7 high-redshift supernovae of the Supernova Cosmology Project (Perlmutter et al. 1997) respectively indicated that $\Omega_{\Lambda}<0.66$ and $\Omega_{\Lambda}<0.51$ at $95 \%$ confidence, for a flat Universe. The discovery of accelerated expansion in 1998 saved inflation by providing evidence for large $\Omega_{\Lambda}$ and was thus welcome news for cosmology.

\subsection{Discovery}

Two breakthroughs enabled the discovery of cosmic acceleration. The first was the demonstration that type Ia supernovae (SNe Ia) are standardizable candles (Phillips 1993). The second was the deployment of large mosaic CCD cameras on 4-meter class telescopes, enabling the systematic search of large areas of sky, containing thousands of galaxies, for these rare events. By comparing deep, wide images taken weeks apart, the discovery of SNe at redshifts $z \sim 0.5$ could be "scheduled" on a statistical basis.

Two teams, the Supernova Cosmology Project and the High-z SN Search, working independently in the mid- to late-1990s took advantage of these breakthroughs to measure the SN Hubble diagram to much larger distances than was previously possible. Both teams found that distant $\mathrm{SNe}$ are $\sim 0.25$ mag dimmer than they would be in a decelerating Universe, indicating that the expansion has been speeding up for the past 5 Gyr (Perlmutter et al. 1999, Riess et al. 1998); see Fig. 4. When analyzed assuming a Universe with matter and cosmological constant, their results provided evidence for $\Omega_{\Lambda}>0$ at greater than $99 \%$ confidence (see Fig. 8 for the current constraints).

\section{CURRENT STATUS}

Since the supernova discoveries were announced in 1998, the evidence for an accelerating Universe has become substantially stronger and more broadly based. 


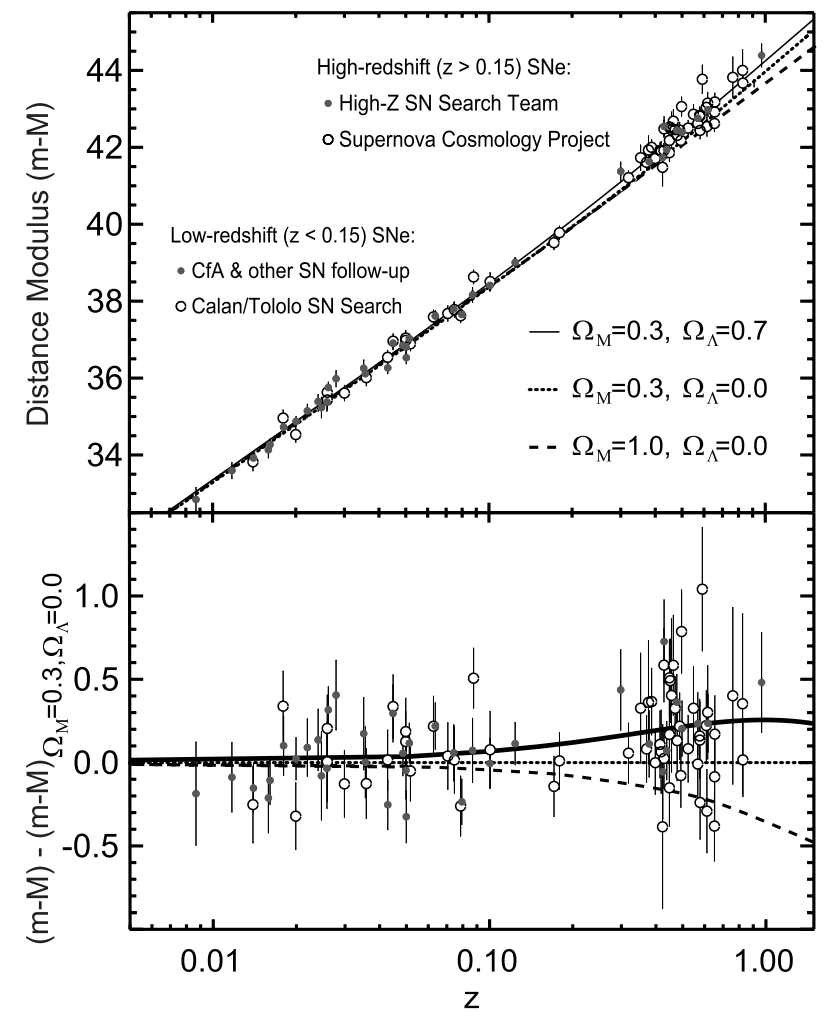

Figure 4: Discovery data: Hubble diagram of SNe Ia measured by the Supernova Cosmology Project and the High-z Supernova Team. Bottom panel shows residuals in distance modulus relative to an open universe with $\Omega_{0}=\Omega_{\mathrm{M}}=0.3$. Figure adapted from Perlmutter \& Schmidt (2003). Riess (2000). based on Perlmutter et al. (1999), Riess et al. (1998).

Subsequent supernova observations have reinforced the original results, and new evidence has accrued from other observational probes. In this section, we review these developments and discuss the current status of the evidence for cosmic acceleration and what we know about dark energy. In \$7, we address the probes of cosmic acceleration in more detail, and we discuss future experiments in $₫ 8$.

\subsection{Cosmic microwave background and large-scale structure}

An early and important confirmation of accelerated expansion was the independent evidence for dark energy from measurements of CMB anisotropy (Jaffe et al. 2001, Pryke et al. 2002) and of large-scale structure (LSS). The CMB constrains the amplitude of the primordial fluctuations that give rise to the observed structure as well as the distance to the last-scattering surface, $r(z \simeq 1100)$. In order to allow sufficient growth of the primordial perturbations and not disrupt the formation of large-scale structure, dark energy must come to dominate the Universe only very recently (see 2.3 ), implying that its energy density must evolve with redshift more slowly than matter. This occurs if it has negative pressure, $w<0$, cf. Eq. (5). Likewise, the presence of a component with large negative pressure that accounts for three-quarters of the critical density affects the distance to the last-scattering surface. 

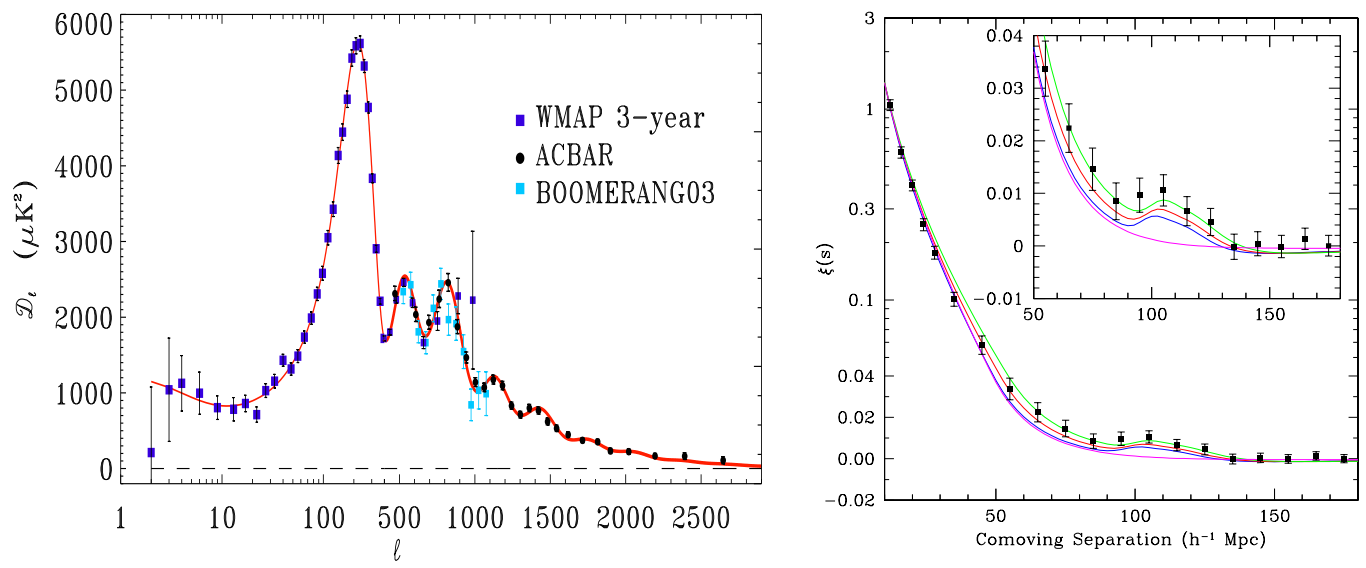

Figure 5: Left panel: Angular power spectrum measurements of the CMB temperature fluctuations from WMAP, Boomerang, and ACBAR. Red curve shows the best-fit $\Lambda$ CDM model. From Reichardt et al. (2008). Right panel: Detection of the baryon acoustic peak in the clustering of luminous red galaxies in the SDSS (Eisenstein et al. 2005). Shown is the two-point galaxy correlation function in redshift space; inset shows an expanded view with a linear vertical axis. Curves correspond to $\Lambda$ CDM predictions for $\Omega_{\mathrm{M}} h^{2}=0.12$ (green), 0.13 (red), and 0.14 (blue). Magenta curve shows a $\Lambda$ CDM model without BAO.

4.1.1 CMB Anisotropies of the cosmic microwave background provide a record of the Universe at a simpler time, before structure had developed and when photons were decoupling from baryons, about 380,000 years after the Big Bang ( $\mathrm{Hu} \&$ Dodelson 2002). The angular power spectrum of CMB temperature anisotropies, measured most recently by WMAP (Spergel et al. 2007) and by ground-based experiments that probe to smaller angular scales, is dominated by acoustic peaks that arise from gravity-driven sound waves in the photonbaryon fluid (see Fig. 5a). The positions and amplitudes of the acoustic peaks encode a wealth of cosmological information. They indicate that the Universe is nearly spatially flat to within a few percent. In combination with LSS or with independent $H_{0}$ measurement, the $\mathrm{CMB}$ measurements indicate that matter contributes only about a quarter of the critical density. A component of missing energy that is smoothly distributed is needed to square these observations - and is fully consistent with the dark energy needed to explain accelerated expansion.

4.1.2 LARGe-SCALE Structure Baryon acoustic oscillations (BAO), so prominent in the $\mathrm{CMB}$ anisotropy, leave a subtler characteristic signature in the clustering of galaxies, a bump in the two-point correlation function at a scale $\sim 100 \mathrm{Mpc}$ that can be measured today and in the future can provide a powerful probe of dark energy (see 97.3 ). Measurement of the BAO signature in the correlation function of SDSS luminous red galaxies (see Fig. 5b) constrains the distance to redshift $z=0.35$ to a precision of $5 \%$ (Eisenstein et al. 2005). This measurement serves as a significant complement to other probes, as shown in Fig. 8 ,

The presence of dark energy affects the large-angle anisotropy of the CMB (the low- $\ell$ multipoles) through the integrated Sachs-Wolfe (ISW) effect. The 
ISW arises due to the differential redshifts of photons as they pass through timechanging gravitational potential wells, and it leads to a small correlation between the low-redshift matter distribution and the CMB anisotropy. This effect has been observed in the cross-correlation of the CMB with galaxy and radio source catalogs (Afshordi. Loh \& Strauss 2004: Boughn \& Crittenden 2004: Fosalba \& Gaztanaga 2004; Scranton et al. 2003). This signal indicates that the Universe is not described by the Einstein-de Sitter model $\left(\Omega_{\mathrm{M}}=1\right)$, a reassuring cross-check.

Weak gravitational lensing (Munshi et al. 2006, Schneider 2006), the small, correlated distortions of galaxy shapes due to gravitational lensing by intervening large-scale structure, is a powerful technique for mapping dark matter and its clustering. Detection of this cosmic shear signal was first announced by four groups in 2000 (Bacon. Refregier \& Ellis 2000; Kaiser. Wilson \& Luppino 2000; Van Waerbeke et al. 2000; Wittman et al. 2000). Recent lensing surveys covering areas of order 100 square degrees have shed light on dark energy by pinning down the combination $\sigma_{8}\left(\Omega_{\mathrm{M}} / 0.25\right)^{0.6} \approx 0.85 \pm 0.07$, where $\sigma_{8}$ is the rms amplitude of mass fluctuations on the $8 h^{-1} \mathrm{Mpc}$ scale (Hoekstra et al. 2006, Jarvis et al. 2006, Massey et al. 2007). Since other measurements peg $\sigma_{8}$ at $\simeq 0.8$, this implies that $\Omega_{\mathrm{M}} \simeq 0.25$, consistent with a flat Universe dominated by dark energy. In the future, weak lensing has the potential to be the most powerful probe of dark energy ( $\mathrm{Hu}$ 2002, Huterer 2002), and this is discussed in 97 and 98 .

\subsection{Recent supernova results}

A number of concerns were raised about the robustness of the first SN evidence for acceleration, e.g., it was suggested that distant SNe could appear fainter due to extinction by hypothetical grey dust rather than acceleration Aguirre 1999; Drell. Loredo \& Wasserman 2000). Over the intervening decade, the supernova evidence for acceleration has been strengthened by results from a series of SN surveys. Observations with the Hubble Space Telescope (HST) have provided high-quality light curves (Knop et al. 2003) and have extended SN measurements to redshift $z \simeq 1.8$, providing evidence for the expected earlier epoch of deceleration and disfavoring dust extinction as an alternative explanation to acceleration (Riess et al. 2001, 2007, Riess et al. 2004). Two large ground-based surveys, the Supernova Legacy Survey (SNLS) (Astier et al. 2006) and the ESSENCE survey (Miknaitis et al. 2007), have been using 4-meter telescopes to measure light curves for several hundred SNe Ia over the redshift range $z \sim 0.3-0.9$, with large programs of spectroscopic follow-up on 6- to 10-m telescopes. Fig. 6 shows a compilation of SN distance measurements from these and other surveys. The quality and quantity of the distant SN data are now vastly superior to what was available in 1998, and the evidence for acceleration is correspondingly more secure (see Fig. 8).

\subsection{X-ray clusters}

Measurements of the ratio of X-ray emitting gas to total mass in galaxy clusters, $f_{\text {gas }}$, also indicate the presence of dark energy. Since galaxy clusters are the largest collapsed objects in the universe, the gas fraction in them is presumed to be constant and nearly equal to the baryon fraction in the Universe, $f_{\text {gas }} \approx \Omega_{\mathrm{B}} / \Omega_{\mathrm{M}}$ (most of the baryons in clusters reside in the gas). The value of $f_{\text {gas }}$ inferred from observations depends on the observed X-ray flux and temperature as well as the 

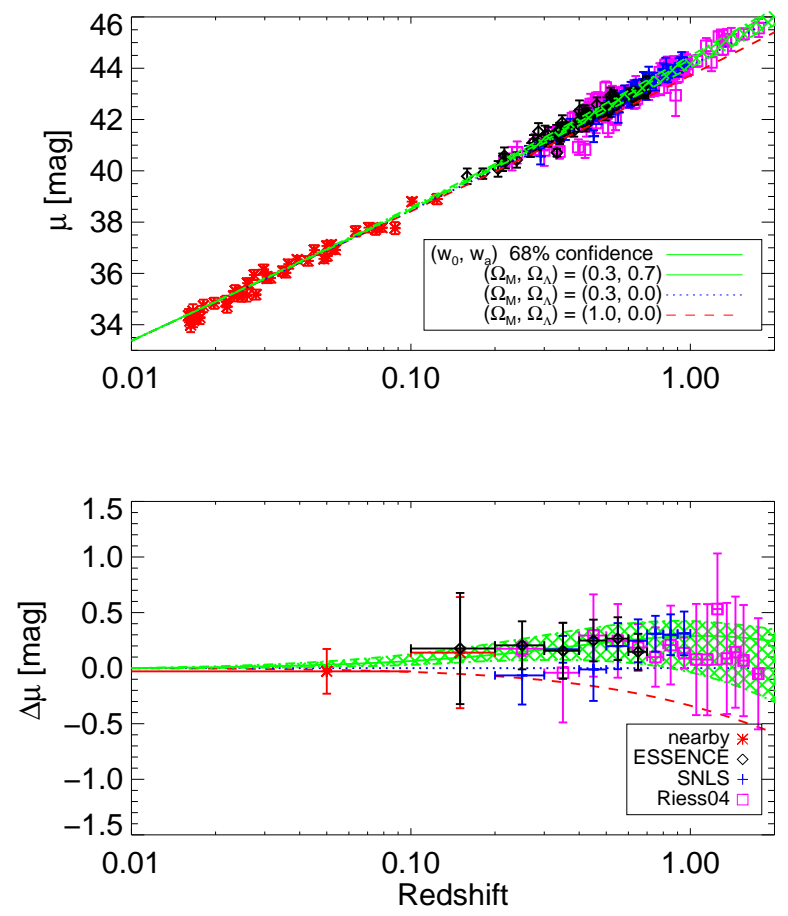

Figure 6: SN Ia results: ESSENCE (diamonds), SNLS (crosses), low-redshift SNe $\left(^{*}\right)$, and the compilation of Riess et al. (2004) which includes many of the other published SN distances plus those from HST (squares). Upper: distance modulus vs. redshift measurements shown with three cosmological models: $\Omega_{\mathrm{M}}=0.3$, $\Omega_{\Lambda}=0$ (dotted); $\Omega_{\mathrm{M}}=1, \Omega_{\Lambda}=0$ (dashed); and the $68 \%$ CL allowed region in the $w_{0}-w_{a}$ plane, assuming spatial flatness and a prior of $\Omega_{\mathrm{M}}=0.27 \pm 0.03$ (hatched). Lower: binned distance modulus residuals from the $\Omega_{\mathrm{M}}=0.3, \Omega_{\Lambda}=0$ model. Adapted from Wood-Vasey et al. (2007).

distance to the cluster. Only the "correct cosmology" will produce distances which make the apparent $f_{\text {gas }}$ constant in redshift. Using data from the Chandra X-ray Observatory, Allen et al. (2007), Allen et al. (2004) determined $\Omega_{\Lambda}$ to a $68 \%$ precision of about \pm 0.2 , obtaining a value consistent with the $\mathrm{SN}$ data.

\subsection{Age of the Universe}

Finally, because the expansion age of the Universe depends upon the expansion history, the comparison of this age with independent age estimates can be used to probe dark energy. The ages of the oldest stars in globular clusters constrain the age of the Universe: 12 Gyr $\lesssim t_{0} \lesssim 15$ Gyr (Krauss \& Chaboyer 2003). When combined with a weak constraint from structure formation or from dynamical measurements of the matter density, $0.2<\Omega_{\mathrm{M}}<0.3$, a consistent age is possible if $-2 \lesssim w \lesssim-0.5$; see Fig. 7 . Age consistency is an important crosscheck and provides additional evidence for the defining feature of dark energy, large negative pressure. CMB anisotropy is very sensitive to the expansion age; in combination with large-scale structure measurements, for a flat Universe it yields the tight constraint $t_{0}=13.8 \pm 0.2$ Gyr (Tegmark et al. 2006). 


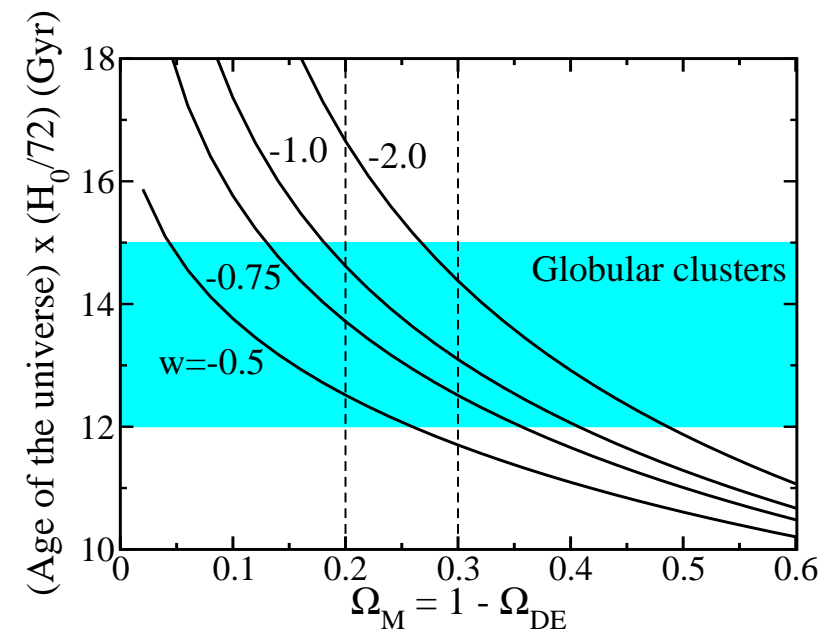

Figure 7: Expansion age of a flat universe vs. $\Omega_{\mathrm{M}}$ for different values of $w$. Shown in blue are age constraints from globular clusters (Krauss \& Chabover 2003), and vertical dashed lines indicate the favored range for $\Omega_{\mathrm{M}}$. Age consistency obtains for $-2 \lesssim w \lesssim-0.5$.

\subsection{Cosmological parameters}

Sandage (1970) once described cosmology as the quest for two numbers, $H_{0}$ and $q_{0}$, which were just beyond reach. Today's cosmological model is described by anywhere from 4 to 20 parameters, and the quantity and quality of cosmological data described above enables precise constraints to be placed upon all of them. However, the results depend on which set of parameters are chosen to describe the Universe as well as the mix of data used.

For definiteness, we refer to the "consensus cosmological model" (or $\Lambda \mathrm{CDM}$ ) as one in which $k, H_{0}, \Omega_{\mathrm{B}}, \Omega_{\mathrm{M}}, \Omega_{\Lambda}, t_{0}, \sigma_{8}$, and $n_{S}$ are free parameters, but dark energy is assumed to be a cosmological constant, $w=-1$. For this model. Tegmark et al. (2006) combined data from SDSS and WMAP to derive the constraints

Table 1: Cosmological parameter constraints from Tegmark et al. (2006).

\begin{tabular}{ccc}
\hline \hline Parameter & Consensus model & Fiducial model \\
\hline$\Omega_{0}$ & $1.003 \pm 0.010$ & 1 (fixed) \\
$\Omega_{\mathrm{DE}}$ & $0.757 \pm 0.021$ & $0.757 \pm 0.020$ \\
$\Omega_{\mathrm{M}}$ & $0.246 \pm 0.028$ & $0.243 \pm 0.020$ \\
$\Omega_{\mathrm{B}}$ & $0.042 \pm 0.002$ & $0.042 \pm 0.002$ \\
$\sigma_{8}$ & $0.747 \pm 0.046$ & $0.733 \pm 0.048$ \\
$n_{S}$ & $0.952 \pm 0.017$ & $0.950 \pm 0.016$ \\
$H_{0}(\mathrm{~km} / \mathrm{s} / \mathrm{Mpc})$ & $72 \pm 5$ & $72 \pm 3$ \\
$T_{0}(\mathrm{~K})$ & $2.725 \pm 0.001$ & $2.725 \pm 0.001$ \\
$t_{0}(\mathrm{Gyr})$ & $13.9 \pm 0.6$ & $13.8 \pm 0.2$ \\
$w$ & $-1($ fixed $)$ & $-0.94 \pm 0.1$ \\
$q_{0}$ & $-0.64 \pm 0.03$ & $-0.57 \pm 0.1$ \\
\hline \hline
\end{tabular}



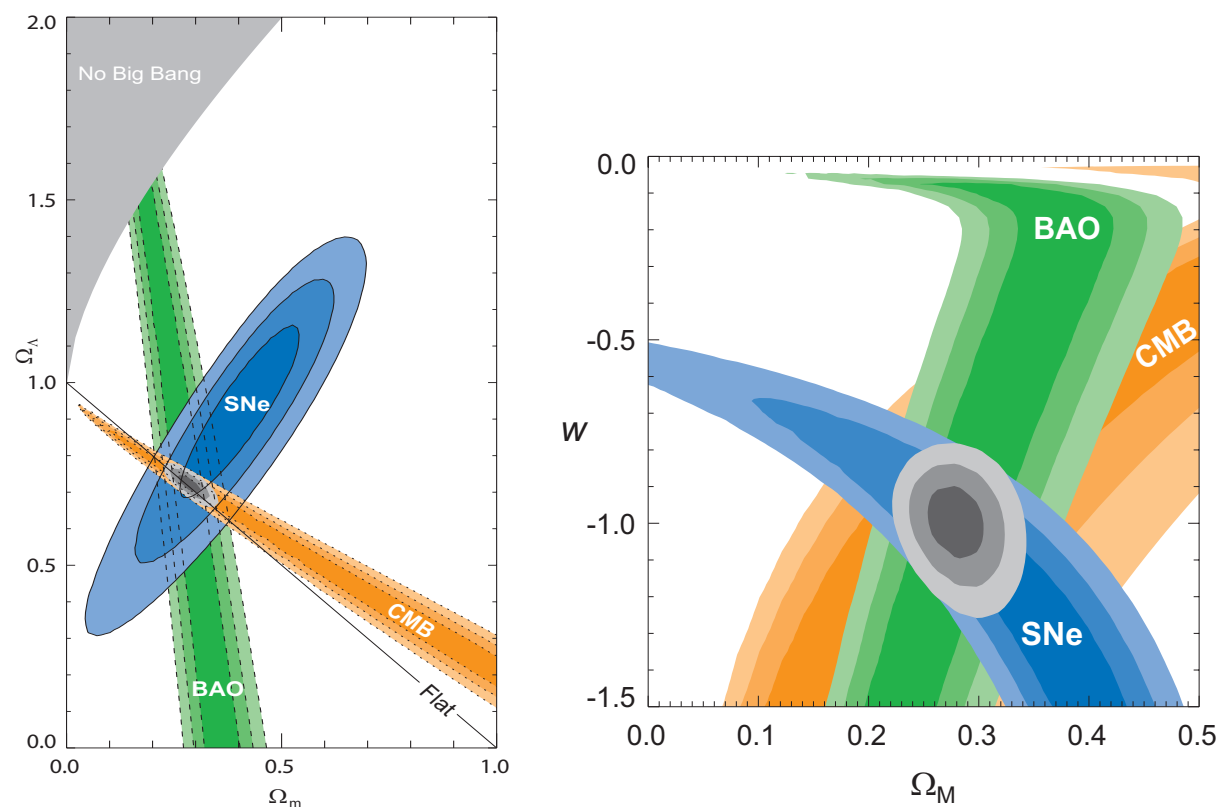

Figure 8: Left panel: Constraints upon $\Omega_{\mathrm{M}}$ and $\Omega_{\Lambda}$ in the consensus model using BAO, CMB, and SNe measurements. Right panel: Constraints upon $\Omega_{\mathrm{M}}$ and constant $w$ in the fiducial dark energy model using the same data sets. From Kowalski et al. (2008).

shown in the second column of Table 1,

To both illustrate and gauge the sensitivity of the results to the choice of cosmological parameters, we also consider a "fiducial dark energy model", in which spatial flatness $\left(k=0, \Omega_{0}=1\right)$ is imposed, and $w$ is assumed to be a constant that can differ from -1 . For this case the cosmological parameter constraints are given in the third column of Table 1 .

Although $w$ is not assumed to be -1 in the fiducial model, the data prefer a value that is consistent with this, $w=-0.94 \pm 0.1$. Likewise, the data prefer spatial flatness in the consensus model in which flatness is not imposed. For the other parameters, the differences are small. Fig. 8 shows how different data sets individually and in combination constrain parameters in these two models; although the mix of data used here differs from that in Table1(SNe are included in Fig. 8), the resulting constraints are consistent.

Regarding Sandage's two numbers, Table 1 reflects good agreement with but a smaller uncertainty than the direct $H_{0}$ measurement based upon the extragalactic distance scale, $H_{0}=72 \pm 8 \mathrm{~km} / \mathrm{s} / \mathrm{Mpc}$ (Freedman et al. 2001). However, the parameter values in Table 1 are predicated on the correctness of the CDM paradigm for structure formation. The entries for $q_{0}$ in Table 1 are derived from the other parameters using Eq. (6). Direct determinations of $q_{0}$ require either ultra-precise distances to objects at low redshift or precise distances to objects at moderate redshift. The former is still beyond reach, while for the latter the $H_{0} / q_{0}$ expansion is not valid.

If we go beyond the restrictive assumptions of these two models, allowing both curvature and $w$ to be free parameters, then the parameter values shift slightly and the errors increase, as expected. In this case, combining WMAP, SDSS, 2dFGRS, and SN Ia data, Spergel et al. (2007) find $w=-1.08 \pm 0.12$ and 


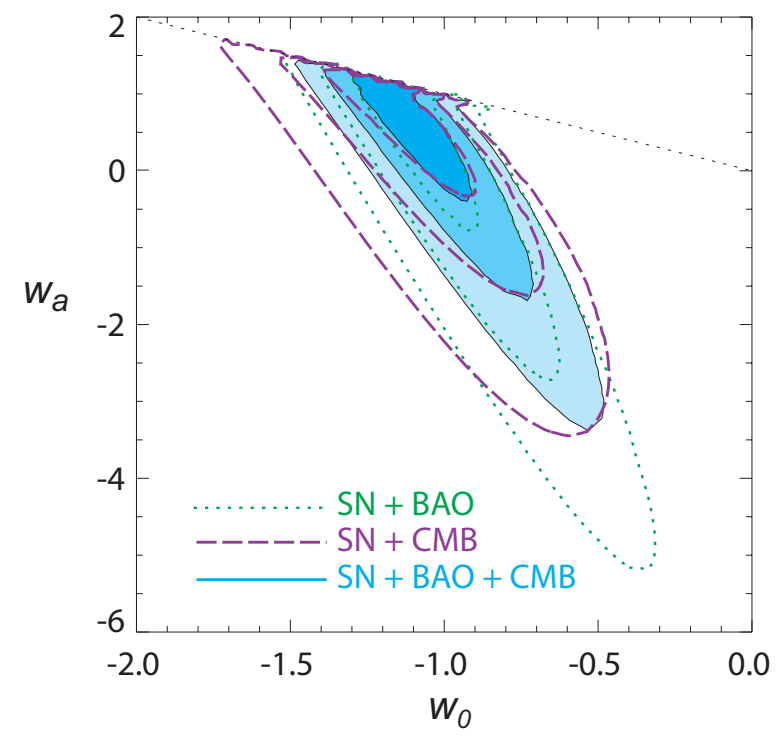

Figure 9: $68.3 \%, 95.4 \%$, and $99.7 \%$ C.L. marginalized constraints on $w_{0}$ and $w_{a}$ in a flat Universe, using data from SNe, CMB, and BAO. The diagonal line indicates $w_{0}+w_{a}=0$. From Kowalski et al. (2008).

$\Omega_{0}=1.026_{-0.015}^{+0.016}$, while WMAP+SDSS only bounds $H_{0}$ to the range $61-84$ $\mathrm{km} / \mathrm{s} / \mathrm{Mpc}$ at $95 \%$ confidence (Tegmark et al.|2006), comparable to the accuracy of the HST Key Project measurement (Freedman et al. 2001).

Once we drop the assumption that $w=-1$, there are no strong theoretical reasons for restricting attention to constant $w$. A widely used and simple form that accommodates evolution is $w=w_{0}+(1-a) w_{a}$ (see $\sqrt[66]{6}$ ). Future surveys with greater reach than that of present experiments will aim to constrain models in which $\Omega_{\mathrm{M}}, \Omega_{\mathrm{DE}}, w_{0}$, and $w_{a}$ are all free parameters (see 98 ). We note that the current observational constraints on such models are quite weak. Fig. 9] shows the marginalized constraints on $w_{0}$ and $w_{a}$ when just three of these four parameters are allowed to vary, using data from the $\mathrm{CMB}, \mathrm{SNe}$, and $\mathrm{BAO}$, corresponding to $w_{0} \simeq-1 \pm 0.2, w_{a} \sim 0 \pm 1$ (Kowalski et al. 2008). While the extant data are fully consistent with $\Lambda C D M$, they do not exclude more exotic models of dark energy in which the dark energy density or its equation-of-state parameter vary with time.

\section{UNDERSTANDING COSMIC ACCELERATION}

Understanding the origin of cosmic acceleration presents a stunning opportunity for theorists. As discussed in $₫ 2$, a smooth component with large negative pressure has repulsive gravity and can lead to the observed accelerated expansion within the context of GR. This serves to define dark energy. There is no shortage of ideas for what dark energy might be, from the quantum vacuum to a new, ultra-light scalar field. Alternatively, cosmic acceleration may arise from new gravitational physics, perhaps involving extra spatial dimensions. Here, we briefly review the theoretical landscape. 


\subsection{Dark energy models}

5.1.1 VACUUM ENERGY Vacuum energy is simultaneously the most plausible and most puzzling dark energy candidate. General covariance requires that the stress-energy of the vacuum takes the form of a constant times the metric tensor, $T_{\mathrm{VAC}}^{\mu \nu}=\rho_{\mathrm{VAC}} g^{\mu \nu}$. Because the diagonal terms $\left(T_{0}^{0}, T_{i}^{i}\right)$ of the stress-energy tensor $T_{\nu}^{\mu}$ are the energy density and minus the pressure of the fluid, and $g_{\nu}^{\mu}$ is just the Kronecker delta, the vacuum has a pressure equal to minus its energy density, $p_{\mathrm{VAC}}=-\rho_{\mathrm{VAC}}$. This also means that vacuum energy is mathematically equivalent to a cosmological constant.

Attempts to compute the value of the vacuum energy density lead to very large or divergent results. For each mode of a quantum field there is a zero-point energy $\hbar \omega / 2$, so that the energy density of the quantum vacuum is given by

$$
\rho_{\mathrm{VAC}}=\frac{1}{2} \sum_{\text {fields }} g_{i} \int_{0}^{\infty} \sqrt{k^{2}+m^{2}} \frac{d^{3} k}{(2 \pi)^{3}} \simeq \sum_{\text {fields }} \frac{g_{i} k_{\text {max }}^{4}}{16 \pi^{2}}
$$

where $g_{i}$ accounts for the degrees of freedom of the field (the sign of $g_{i}$ is + for bosons and - for fermions), and the sum runs over all quantum fields (quarks, leptons, gauge fields, etc). Here $k_{\max }$ is an imposed momentum cutoff, because the sum diverges quartically.

To illustrate the magnitude of the problem, if the energy density contributed by just one field is to be at most the critical density, then the cutoff $k_{\max }$ must be $<0.01 \mathrm{eV}$ - well below any energy scale where one could have appealed to ignorance of physics beyond. [Pauli apparently carried out this calculation in the 1930's, using the electron mass scale for $k_{\max }$ and finding that the size of the Universe, that is, $H^{-1}$, "could not even reach to the moon" (Straumann 2002).] Taking the cutoff to be the Planck scale $\left(\approx 10^{19} \mathrm{GeV}\right)$, where one expects quantum field theory in a classical spacetime metric to break down, the zero-point energy density would exceed the critical density by some 120 orders-of-magnitude! It is very unlikely that a classical contribution to the vacuum energy density would cancel this quantum contribution to such high precision. This very large discrepancy is known as the cosmological constant problem (Weinberg 1989).

Supersymmetry, the hypothetical symmetry between bosons and fermions, appears to provide only partial help. In a supersymmetric (SUSY) world, every fermion in the standard model of particle physics has an equal-mass SUSY bosonic partner and vice versa, so that fermionic and bosonic zero-point contributions to $\rho_{\mathrm{VAC}}$ would exactly cancel. However, SUSY is not a manifest symmetry in Nature: none of the SUSY particles has yet been observed in collider experiments, so they must be substantially heavier than their standard-model partners. If SUSY is spontaneously broken at a mass scale $M$, one expects the imperfect cancellations to generate a finite vacuum energy density $\rho_{\mathrm{VAC}} \sim M^{4}$. For the currently favored value $M \sim 1 \mathrm{TeV}$, this leads to a discrepancy of 60 (as opposed to 120) orders of magnitude with observations. Nonetheless, experiments at the Large Hadron Collider (LHC) at CERN will soon begin searching for signs of SUSY, e.g., SUSY partners of the quarks and leptons, and might shed light on the vacuum-energy problem.

Another approach to the cosmological constant problem involves the idea that the vacuum energy scale is a random variable that can take on different values in different disconnected regions of the Universe. Because a value much larger than that needed to explain the observed cosmic acceleration would preclude 


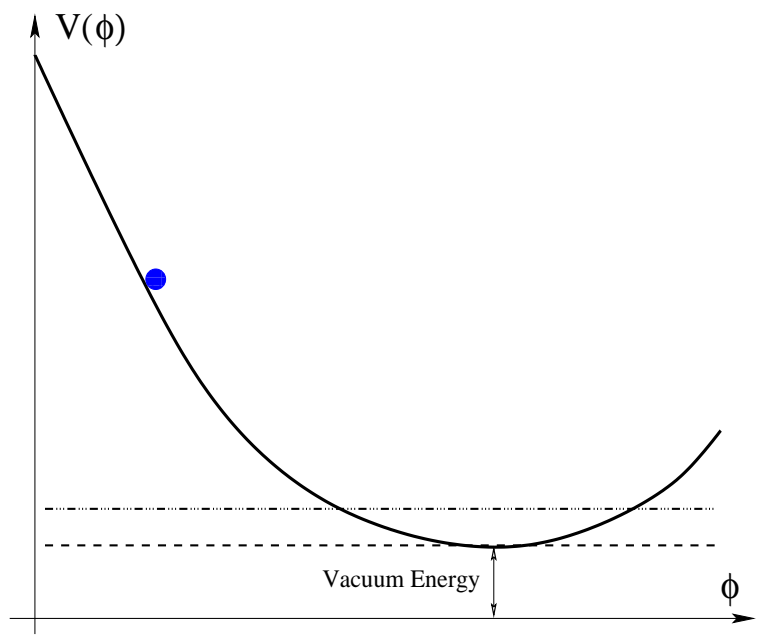

Figure 10: Generic scalar potential $V(\phi)$. The scalar field rolls down the potential eventually settling at its minimum, which corresponds to the vacuum. The energy associated with the vacuum can be positive, negative, or zero.

the formation of galaxies (assuming all other cosmological parameters are held fixed), we could not find ourselves in a region with such large $\rho_{\mathrm{VAC}}$ (Weinberg 1987). This anthropic approach finds a possible home in the landscape version of string theory, in which the number of different vacuum states is very large and essentially all values of the cosmological constant are possible. Provided that the Universe has such a multiverse structure, this might provide an explanation for the smallness of the cosmological constant (Bousso \& Polchinski 2000, Susskind 2003).

5.1.2 SCAlAR FIELDS Vacuum energy does not vary with space or time and is not dynamical. However, by introducing a new degree of freedom, a scalar field $\phi$, one can make vacuum energy effectively dynamical (Frieman et al. 1995; Ratra \& Peebles 1988; Wetterich 1988; Zlatev, Wang \& Steinhardt 1999). For a scalar field $\phi$, with Lagrangian density $\mathcal{L}=\frac{1}{2} \partial^{\mu} \phi \partial_{\mu} \phi-V(\phi)$, the stress-energy takes the form of a perfect fluid, with

$$
\rho=\dot{\phi}^{2} / 2+V(\phi), \quad p=\dot{\phi}^{2} / 2-V(\phi),
$$

where $\phi$ is assumed to be spatially homogeneous, i.e., $\phi(\vec{x}, t)=\phi(t), \dot{\phi}^{2} / 2$ is the kinetic energy, and $V(\phi)$ is the potential energy; see Fig. 10, The evolution of the field is governed by its equation of motion,

$$
\ddot{\phi}+3 H \dot{\phi}+V^{\prime}(\phi)=0,
$$

where a prime denotes differentiation with respect to $\phi$. Scalar-field dark energy can be described by the equation-of-state parameter

$$
w=\frac{\dot{\phi}^{2} / 2-V(\phi)}{\dot{\phi}^{2} / 2+V(\phi)}=\frac{-1+\dot{\phi}^{2} / 2 V}{1+\dot{\phi}^{2} / 2 V} .
$$

If the scalar field evolves slowly, $\dot{\phi}^{2} / 2 V \ll 1$, then $w \approx-1$, and the scalar field behaves like a slowly varying vacuum energy, with $\rho_{\mathrm{VAC}}(t) \simeq V[\phi(t)]$. In general, 
from Eq. (20), $w$ can take on any value between -1 (rolling very slowly) and +1 (evolving very rapidly) and varies with time.

Many scalar field models can be classified dynamically as "thawing" or "freezing" (Caldwell \& Linder 2005). In freezing models, the field rolls more slowly as time progresses, i.e., the slope of the potential drops more rapidly than the Hubble friction term $3 H \dot{\phi}$ in Eq. (19). This can happen if, e.g., $V(\phi)$ falls off exponentially or as an inverse power-law at large $\phi$. For thawing models, at early times the field is frozen by the friction term, and it acts as vacuum energy; when the expansion rate drops below $H^{2}=V^{\prime \prime}(\phi)$, the field begins to roll and $w$ evolves away from -1 . The simplest example of a thawing model is a scalar field of mass $m_{\phi}$, with $V(\phi)=m_{\phi}^{2} \phi^{2} / 2$. Since thawing and freezing fields tend to have different trajectories of $w(z)$, precise cosmological measurements might be able to discriminate between them.

5.1.3 Cosmic coincidence and Scalar Fields As Fig. 1 1 shows, through most of the history of the Universe, dark matter or radiation dominated dark energy by many orders of magnitude. We happen to live around the time that dark energy has become important. Is this coincidence between $\rho_{\mathrm{DE}}$ and $\rho_{\mathrm{M}}$ an important clue to understanding cosmic acceleration or just a natural consequence of the different scalings of cosmic energy densities and the longevity of the Universe? In some freezing models, the scalar field energy density tracks that of the dominant component (radiation or matter) at early times and then dominates at late times, providing a dynamical origin for the coincidence. In thawing models, the coincidence is indeed transitory and just reflects the mass scale of the scalar field.

5.1.4 More COMPliCATED SCALAR-FIELD MOdELS While the choice of the potential $V(\phi)$ allows a large range of dynamical behaviors, theorists have also considered the implications of modifying the canonical form of the kinetic energy term $\frac{1}{2} \partial^{\mu} \phi \partial_{\mu} \phi$ in the Lagrangian. By changing the sign of this term, from Eq. 20 it is possible to have $w<-1$ (Caldwell 2002), although such theories are typically unstable (Carroll. Hoffman \& Trodden 2003). In "k-essence," one introduces a field-dependent kinetic term in the Lagrangian to address the coincidence problem (Armendariz-Picon, Mukhanov \& Steinhardt 2000).

5.1.5 SCALAR-FIELD ISSUES Scalar-field models raise new questions and possibilities. For example, is cosmic acceleration related to inflation? After all, both involve accelerated expansion and can be explained by scalar field dynamics. Is dark energy related to dark matter or neutrino mass? No firm or compelling connections have been made to either, although the possibilities are intriguing. Unlike vacuum energy, which must be spatially uniform, scalar-field dark energy can clump, providing a possible new observational feature, but in most cases is only expected to do so on the largest observable scales today (see §10.2.1).

Introducing a new dynamical degree of freedom allows for a richer variety of explanations for cosmic acceleration, but it is not a panacea. Scalar field models do not address the cosmological constant problem: they simply assume that the minimum value of $V(\phi)$ is very small or zero; see Fig. 10. Cosmic acceleration is then attributable to the fact that the Universe has not yet reached its true vacuum state, for dynamical reasons. These models also pose new challenges: in order to roll slowly enough to produce accelerated expansion, the effective mass of the scalar field must be very light compared to other mass scales in particle physics, $m_{\phi} \equiv \sqrt{V^{\prime \prime}(\phi)} \lesssim 3 H_{0} \simeq 10^{-42} \mathrm{GeV}$, even though the field amplitude 
is typically of order the Planck scale, $\phi \sim 10^{19} \mathrm{GeV}$. This hierarchy, $m_{\phi} / \phi \sim$ $10^{-60}$, means that the scalar field potential must be extremely flat. Moreover, in order not to spoil this flatness, the interaction strength of the field with itself must be extremely weak, at most of order $10^{-120}$ in dimensionless units; its coupling to matter must also be very weak to be consistent with constraints upon new long-range forces (Carroll 1998). Understanding such very small numbers and ratios makes it challenging to connect scalar field dark energy with particle physics models (Frieman et al. 1995). In constructing theories that go beyond the standard model of particle physics, including those that incorporate primordial inflation, model-builders have been strongly guided by the requirement that any small dimensionless numbers in the theory should be protected by symmetries from large quantum corrections (as in the SUSY example above). Thus far, such model-building discipline has not been the rule among cosmologists working on dark energy models.

\subsection{Modified gravity}

A very different approach holds that cosmic acceleration is a manifestation of new gravitational physics rather than dark energy, i.e., that it involves a modification of the geometric as opposed to the stress-tensor side of the Einstein equations. Assuming that 4-d spacetime can still be described by a metric, the operational changes are twofold: (1) a new version of the Friedmann equation governing the evolution of $a(t)$; (2) modifications to the equations that govern the growth of the density perturbations that evolve into large-scale structure. A number of ideas have been explored along these lines, from models motivated by higher-dimensional theories and string theory (Deffavet 2001: Dvali, Gabadadze \& Porrati 2000) to phenomenological modifications of the Einstein-Hilbert Lagrangian of GR (Carroll et al. 2004; Song, Hu \& Sawicki 2007).

Changes to the Friedmann equation are easier to derive, discuss, and analyze. In order not to spoil the success of the standard cosmology at early times (from big bang nucleosynthesis to the $\mathrm{CMB}$ anisotropy to the formation of structure), the Friedmann equation must reduce to the GR form for $z \gg 1$. As a specific example, consider the model of Dvali, Gabadadze \& Porrati (2000), which arises from a five-dimensional gravity theory and has a 4-d Friedmann equation,

$$
H^{2}=\frac{8 \pi G \rho}{3}+\frac{H}{r_{c}}
$$

where $r_{c}$ is a length scale related to the 5-dimensional gravitational constant. As the energy density in matter and radiation, $\rho$, becomes small, there is an accelerating solution, with $H=1 / r_{c}$. From the viewpoint of expansion, the additional term in the Friedmann equation has the same effect as dark energy that has an equation-of-state parameter which evolves from $w=-1 / 2($ for $z \gg 1$ ) to $w=-1$ in the distant future. While attractive, it is not clear that a consistent model with this dynamical behavior exists (e.g., Gregory et al. 2007).

\subsection{Unmodified gravity}

Instead of modifying the right or left side of the Einstein equations to explain the supernova observations, a third logical possibility is to drop the assumption that the Universe is spatially homogeneous on large scales. It has been argued that 
the non-linear gravitational effects of spatial density perturbations, when averaged over large scales, could yield a distance-redshift relation in our observable patch of the Universe that is very similar to that for an accelerating, homogeneous Universe (Kolb, Matarrese \& Riotto 2006), obviating the need for either dark energy or modified gravity. While there has been debate about the amplitude of these effects, this idea has helped spark renewed interest in a class of exact, inhomogeneous cosmologies. For such Lemaître-Tolman-Bondi models to be consistent with the SN data and not conflict with the isotropy of the CMB, the Milky Way must be near the center of a very large-scale, nearly spherical, underdense region (Alnes. Amarzguioui \& Gron 2006; Enqvist 2007; Tomita 2001). Whether or not such models can be made consistent with the wealth of precision cosmological data remains to be seen; moreover, requiring our galaxy to occupy a privileged location, in violation of the spirit of the Copernican principle, is not yet theoretically well-motivated.

\subsection{Theory summary}

There is no compelling explanation for cosmic acceleration, but many intriguing ideas are being explored. Here is our assessment:

- Cosmological constant: Simple, but no underlying physics

- Vacuum energy: Well-motivated, mathematically equivalent to a cosmological constant; $w=-1$ is consistent with all data, but all attempts to estimate its size are at best orders of magnitude too large

- Scalar fields: Temporary period of cosmic acceleration, $w$ varies between -1 and 1 (and could also be $<-1$ ), possibly related to inflation, but does not address the cosmological constant problem and may lead to new long-range forces

- New gravitational physics: Cosmic acceleration could be a clue to going beyond GR, but no self-consistent model has been put forth

- Old gravitational physics: It may be possible to find an inhomogeneous solution that is observationally viable, but such solutions do not yet seem compelling

The ideas underlying many of these approaches, from attempting to explain the smallness of quantum vacuum energy to extending Einstein's theory, are bold. Solving the puzzle of cosmic acceleration thus has the potential to advance our understanding of many important problems in fundamental physics.

\section{DESCRIBING DARK ENERGY}

The absence of a consensus model for cosmic acceleration presents a challenge in trying to connect theory with observations. For dark energy, the equationof-state parameter $w$ provides a useful phenomenological description (Turner \& White 1997). Because it is the ratio of pressure to energy density, it is also closely connected to the underlying physics. However, $w$ is not fundamentally a function of redshift, and if cosmic acceleration is due to new gravitational physics, the motivation for a description in terms of $w$ disappears. In this section, we review the variety of formalisms that have been used to describe and constrain dark energy. 


\subsection{Parametrizations}

The simplest parameterization of dark energy is $w=$ const. This form fully describes vacuum energy $(w=-1)$ and, together with $\Omega_{\mathrm{DE}}$ and $\Omega_{\mathrm{M}}$, provides a 3 -parameter description of the dark-energy sector (2 parameters if flatness is assumed). However, it does not describe scalar field or modified gravity models.

A number of two-parameter descriptions of $w$ have been explored, e.g., $w(z)=$ $w_{0}+w^{\prime} z$ and $w(z)=w_{0}+b \ln (1+z)$. For low redshift they are all essentially equivalent, but for large $z$, some lead to unrealistic behavior, e.g., $w \ll-1$ or $\gg 1$. The parametrization

$$
w(a)=w_{0}+w_{a}(1-a)=w_{0}+w_{a} z /(1+z)
$$

(e.g., Linder 2003) avoids this problem and leads to the most commonly used description of dark energy, namely $\left(\Omega_{\mathrm{DE}}, \Omega_{\mathrm{M}}, w_{0}, w_{a}\right)$

More general expressions have been proposed, for example, Padé approximants or the transition between two asymptotic values $w_{0}\left(\right.$ at $z \rightarrow 0$ ) and $w_{f}$ (at $z \rightarrow \infty), w(z)=w_{0}+\left(w_{f}-w_{0}\right) /\left(1+\exp \left[\left(z-z_{t}\right) / \Delta\right]\right)$ (Corasaniti \& Copeland 2003).

The two-parameter descriptions of $w(z)$ that are linear in the parameters entail the existence of a "pivot" redshift $z_{p}$ at which the measurements of the two parameters are uncorrelated and the error in $w_{p} \equiv w\left(z_{p}\right)$ reaches a minimum (Huterer \& Turner 2001); see the left panel of Fig. 11. The redshift of this sweet spot varies with the cosmological probe and survey specifications; for example, for current SN Ia surveys $z_{p} \approx 0.25$. Note that forecast constraints for a particular experiment on $w_{p}$ are numerically equivalent to constraints one would derive on constant $w$.

\subsection{Direct reconstruction}

Another approach is to directly invert the redshift-distance relation $r(z)$ measured from SN data to obtain the redshift dependence of $w(z)$ in terms of the first and second derivatives of the comoving distance (Huterer \& Turner 1999, Starobinsky 1998),

$$
1+w(z)=\frac{1+z}{3} \frac{3 H_{0}^{2} \Omega_{\mathrm{M}}(1+z)^{2}+2\left(d^{2} r / d z^{2}\right) /(d r / d z)^{3}}{H_{0}^{2} \Omega_{\mathrm{M}}(1+z)^{3}-(d r / d z)^{-2}} .
$$

Assuming that dark energy is due to a single rolling scalar field, the scalar potential can also be reconstructed,

$$
V[\phi(z)]=\frac{1}{8 \pi G}\left[\frac{3}{(d r / d z)^{2}}+(1+z) \frac{d^{2} r / d z^{2}}{(d r / d z)^{3}}\right]-\frac{3 \Omega_{\mathrm{M}} H_{0}^{2}(1+z)^{3}}{16 \pi G}
$$

Others have suggested reconstructing the dark energy densitv (Wang \& Mukherjee 2004),

$$
\rho_{\mathrm{DE}}(z)=\frac{3}{8 \pi G}\left[\frac{1}{(d r / d z)^{2}}-\Omega_{\mathrm{M}} H_{0}^{2}(1+z)^{3}\right] .
$$

Direct reconstruction is the only approach that is truly model-independent. However, it comes at a price - taking derivatives of noisy data. In practice, one must fit the distance data with a smooth function - e.g., a polynomial, Padé approximant, or spline with tension, and the fitting process introduces systematic biases. While a variety of methods have been pursued (e.g., Gerke \& 

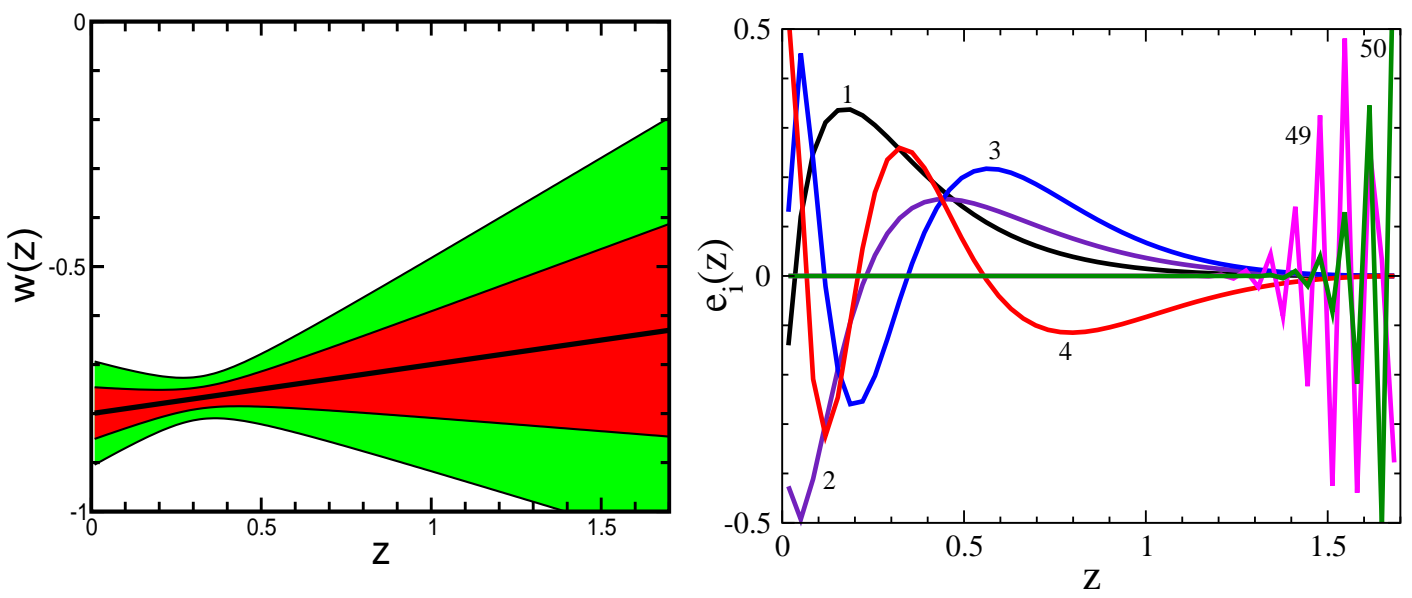

Figure 11: Left panel: Example of forecast constraints on $w(z)$, assuming $w(z)=$ $w_{0}+w^{\prime} z$. The "pivot" redshift, $z_{p} \simeq 0.3$, is where $w(z)$ is best determined. From Huterer \& Turner (2001). Right panel: The four best-determined (labelled 1-4) and two worst-determined (labelled 49,50) principal components of $w(z)$ for a future SN Ia survey such as SNAP, with several thousand SNe in the redshift range $z=0$ to $z=1.7$. From Huterer \& Starkman (2003).

Efstathiou 2002, Weller \& Albrecht 2002), it appears that direct reconstruction is too challenging and not robust even with SN Ia data of excellent quality. Although the expression for $\rho_{\mathrm{DE}}(z)$ involves only first derivatives of $r(z)$, it contains little information about the nature of dark energy. For a review of dark energy reconstruction and related issues, see Sahni \& Starobinsky (2006).

\subsection{Principal components}

The cosmological function that we are trying to determine $-w(z), \rho_{\mathrm{DE}}(z)$, or $H(z)$ - can be expanded in terms of principal components, a set of functions that are uncorrelated and orthogonal by construction (Huterer \& Starkman 2003). In this approach, the data determine which components are measured best.

For example, suppose we parametrize $w(z)$ in terms of piecewise constant values $w_{i}(i=1, \ldots, N)$, each defined over a small redshift range $\left(z_{i}, z_{i}+\Delta z\right)$. In the limit of small $\Delta z$ this recovers the shape of an arbitrary dark energy history (in practice, $N \gtrsim 20$ is sufficient), but the estimates of the $w_{i}$ from a given dark energy probe will be very noisy for large $N$. Principal Component Analysis extracts from those noisy estimates the best-measured features of $w(z)$. We find the eigenvectors $e_{i}(z)$ of the inverse covariance matrix for the parameters $w_{i}$ and the corresponding eigenvalues $\lambda_{i}$. The equation-of-state parameter is then expressed as

$$
w(z)=\sum_{i=1}^{N} \alpha_{i} e_{i}(z),
$$

where the $e_{i}(z)$ are the principal components. The coefficients $\alpha_{i}$, which can be computed via the orthonormality condition, are each determined with an accuracy 
$1 / \sqrt{\lambda_{i}}$. Several of these components are shown for a future SN survey in the right panel of Fig. 11,

One can use this approach to design a survey that is most sensitive to the dark energy equation-of-state parameter in some specific redshift interval or to study how many independent parameters are measured well by a combination of cosmological probes. There are a variety of extensions of this method, including measurements of the equation-of-state parameter in redshift intervals (Huterer \& Cooray 2005).

\subsection{Kinematic description}

If the explanation of cosmic acceleration is a modification of GR and not dark energy, then a purely kinematic description through, e.g., the functions $a(t), H(z)$, or $q(z)$ may be the best approach. With the weaker assumption that gravity is described by a metric theory and that spacetime is isotropic and homogeneous, the FRW metric is still valid, as are the kinematic equations for redshift/scale factor, age, $r(z)$, and volume element. The dynamical equations, i.e., the Friedmann equations and the growth of density perturbations, may however be different.

If $H(z)$ is chosen as the kinematic variable, then $r(z)$ and age take their standard forms. On the other hand, to describe acceleration one might wish to take the deceleration parameter $q(z)$ as the fundamental variable; the expansion rate is then given by

$$
H(z)=H_{0} \exp \left[\int_{0}^{z}\left[1+q\left(z^{\prime}\right)\right] d \ln \left(1+z^{\prime}\right)\right] .
$$

Another possibility is the dimensionless "jerk" parameter, $j \equiv(\dddot{a} / a) / H^{3}$, instead of $q(z)$ (Rapetti et al. 2007, Visser 2004). The deceleration $q(z)$ can be expressed in terms of $j(z)$,

$$
\frac{d q}{d \ln (1+z)}+q(2 q+1)-j=0
$$

and, supplemented by Eq. (27), $H(z)$ may be obtained. Jerk has the virtue that constant $j=1$ corresponds to a cosmology that transitions from $a \propto t^{2 / 3}$ at early times to $a \propto e^{H t}$ at late times. Moreover, for constant jerk, Eq. (28) is easily solved:

$$
\ln \left[\frac{q-q_{+}}{q-q_{-}}\right]=\exp \left[-2\left(q_{+}-q_{-}\right)(1+z)\right], \quad q_{ \pm}=\frac{1}{4}(-1 \pm \sqrt{1+8 j}) .
$$

On the other hand, constant jerk does not span cosmology model-space well: the asymptotic values of deceleration are $q=q_{ \pm}$, so that only for $j=1$ can there be a matter-dominated beginning $\left(q=\frac{1}{2}\right)$. One would test for departures from $\Lambda$ CDM by searching for variation of $j(z)$ from unity over some redshift interval; in principle, the same information is also encoded in $q(z)$.

The kinematic approach has produced some interesting results; using the SN data and the principal component method, Shapiro \& Turner (2006) find the best measured mode of $q(z)$ can be used to infer 5 - $\sigma$ evidence for acceleration of the Universe at some recent time, without recourse to GR and the Friedmann equation. 


\section{PROBES OF COSMIC ACCELERATION}

As described in $\$ 4$, the phenomenon of accelerated expansion is now well established, and the dark energy density has been determined to a precision of a few percent. However, getting at the nature of the dark energy-by measuring its equation-of-state parameter - is more challenging. To illustrate, consider that for fixed $\Omega_{\mathrm{DE}}$, a $1 \%$ change in (constant) $w$ translates to only a $3 \%(0.3 \%)$ change in dark-energy (total) density at redshift $z=2$ and only a $0.2 \%$ change in distances to redshifts $z=1-2$.

The primary effect of dark energy is on the expansion rate of the Universe; in turn, this affects the redshift-distance relation and the growth of structure. While dark energy has been important at recent epochs, we expect that its effects at high redshift were very small, since otherwise it would have been difficult for large-scale structure to have formed (in most models). Since $\rho_{\mathrm{DE}} / \rho_{\mathrm{M}} \propto$ $(1+z)^{3 w} \sim 1 /(1+z)^{3}$, the redshifts of highest leverage for probing dark energy are expected to be between a few tenths and two (Huterer \& Turner 2001). Four methods hold particular promise in probing dark energy in this redshift range: type Ia supernovae, clusters of galaxies, baryon acoustic oscillations, and weak gravitational lensing. In this section, we describe and compare these four probes, highlighting their complementarity in terms of both dark energy constraints and the systematic errors to which they are susceptible. Because of this complementarity, a multi-pronged approach will be most effective. The goals of the next generation of dark energy experiments, described in 8 , are to constrain $w_{0}$ at the few percent level and $w_{a}$ at the $10 \%$ level.

While our focus is on these four techniques, we also briefly discuss other darkenergy probes, emphasizing the important supporting role of the CMB.

\subsection{Supernovae}

By providing bright, standardizable candles (Leibundgut 2001), type Ia supernovae constrain cosmic acceleration through the Hubble diagram, cf., Eq. (11). The first direct evidence for cosmic acceleration came from SNe Ia, and they have provided the strongest constraints on the dark energy equation-of-state parameter. At present, they are the most effective and mature probe of dark energy.

SN Ia light curves are powered by the radioactive decays of ${ }^{56} \mathrm{Ni}$ (at early times) and ${ }^{56} \mathrm{Co}$ (after a few weeks), produced in the thermonuclear explosion of a carbon-oxygen white dwarf accreting mass from a companion star as it approaches the Chandrasekhar mass (Hillebrandt \& Niemever 2000). The peak luminosity is determined by the mass of ${ }^{56} \mathrm{Ni}$ produced in the explosion (Arnett 1982): if the white dwarf is fully burned, one expects $\sim 0.6 M_{\odot}$ of ${ }^{56} \mathrm{Ni}$ to be produced. As a result, although the detailed mechanism of SN Ia explosions remains uncertain (e.g., Hoeflich 2004; Plewa. Calder \& Lamb 2004), SNe Ia are expected to have similar peak luminosities. Since they are about as bright as a typical galaxy when they peak, SNe Ia can be observed to large distances, recommending their utility as standard candles for cosmology.

In fact, as Fig. 12 shows, SNe Ia are not intrinsically standard candles, with a $1 \sigma$ spread of order $0.3 \mathrm{mag}$ in peak $B$-band luminosity which would limit their utility. However, work in the early 1990's (Phillips 1993) established an empirical correlation between SN Ia peak brightness and the rate at which the luminosity declines with time after peak: intrinsically brighter SNe Ia decline 

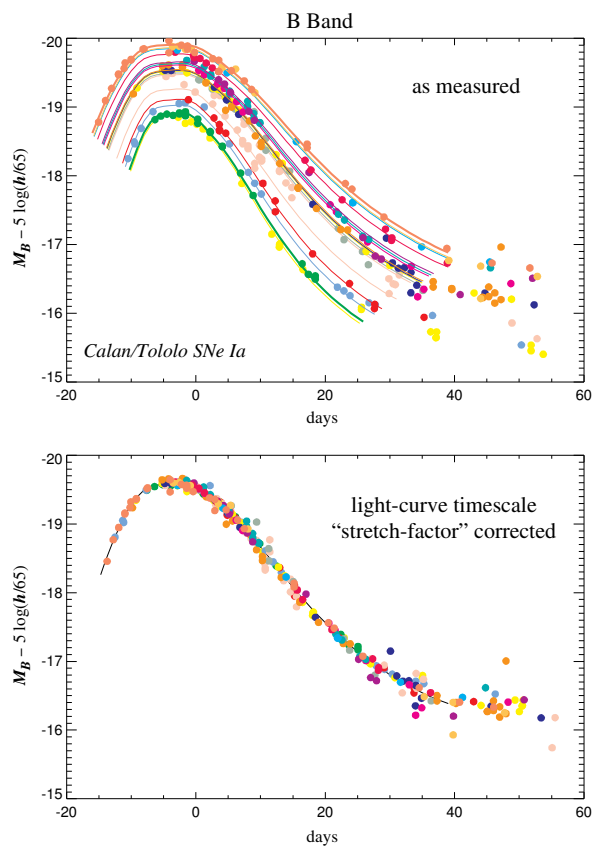

Figure 12: Top panel: B-band light curves for low-redshift SNe Ia from the Calan-Tololo survey (Hamuy et al. 1996) show an intrinsic scatter of $\sim 0.3$ mag in peak luminosity. Bottom panel: After a one-parameter correction for the brightness-decline correlation, the light curves show an intrinsic dispersion of only $\sim 0.15$ mag. From Kim (2004).

more slowly. After correcting for this correlation, SNe Ia turn out to be excellent "standardizable" candles, with a dispersion of about 15\% in peak brightness.

Cosmological parameters are constrained by comparing distances to low- and high-redshift SNe Ia. Operationally, since $H_{0} d_{L}$ is independent of the Hubble parameter $H_{0}$, Eq. (11) can be written as $m=5 \log _{10}\left[H_{0} d_{L}\left(z ; \Omega_{\mathrm{M}}, \Omega_{\mathrm{DE}}, w(z)\right)\right]+$ $\mathcal{M}$, where $\mathcal{M} \equiv M-5 \log _{10}\left(H_{0} \mathrm{Mpc}\right)+25$ is the parameter effectively constrained by the low-redshift SNe that anchor the Hubble diagram.

The major systematic concerns for supernova distance measurements are errors in correcting for host-galaxy extinction and uncertainty in the intrinsic colors of SNe Ia; luminosity evolution; and selection bias in the low-redshift sample. For observations in two passbands, with perfect knowledge of intrinsic SN colors or of the extinction law, one could solve for the extinction and eliminate its effects on the distance modulus. In practice, the combination of photometric errors, variations in intrinsic SN colors, and uncertainties and likely variations in host-galaxy dust properties lead to distance uncertainties even for multi-band observations of SNe. Observations that extend into the rest-frame near-infrared, where the effects of extinction are much reduced, offer promise in controlling this systematic.

With respect to luminosity evolution, there is evidence that SN peak luminosity correlates with host-galaxy type (e.g., Jha, Riess \& Kirshner 2007), and that the mean host-galaxy environment, e.g., the star formation rate, evolves strongly with look-back time. However, brightness-decline-corrected SN Ia Hubble diagrams are consistent between different galaxy types, and since the nearby Universe spans the 
range of galactic environments sampled by the high-redshift SNe, one can measure distances to high-redshift events by comparing with low-redshift analogs. While $\mathrm{SNe}$ provide a number of correlated observables (multi-band light curves and multi-epoch spectra) to constrain the physical state of the system, insights from SN Ia theory will likely be needed to determine if they are collectively sufficient to constrain the mean peak luminosity at the percent level (Hoeflich 2004).

Finally, there is concern that the low-redshift SNe currently used to anchor the Hubble diagram and that serve as templates for fitting distant SN light curves are a relatively small, heterogeneously selected sample and that correlated large-scale peculiar velocities induce larger distance errors than previously estimated (Hui \& Greene 2006). This situation should improve in the near future once results are collected from low-redshift SN surveys such as the Lick Observatory Supernova Search (LOSS), the Center for Astrophysics Supernova project, the Carnegie Supernova Project, the Nearby Supernova Factory, and the Sloan Digital Sky Survey-II Supernova Survey.

Accounting for systematic errors, precision measurement of $w_{0}$ and $w_{a}$ with SNe will require a few thousand SN Ia light curves out to redshifts $z \sim 1.5$ to be measured with unprecedented precision and control of svstematics (Frieman et al. 2003). For redshifts $z>0.8$, this will require going to space to minimize photometric errors, to obtain uniform light-curve coverage, and to observe in the near-infrared bands to capture the redshifted photons.

\subsection{Clusters}

Galaxy clusters are the largest virialized objects in the Universe. Within the context of the CDM paradigm, the number density of cluster-sized dark matter halos as a function of redshift and halo mass can be accurately predicted from N-body simulations (Warren et al. 2006). Comparing these predictions to largearea cluster surveys that extend to high redshift $(z \geq 1)$ can provide precise constraints on the cosmic expansion history (Haiman. Mohr \& Holder 2001: Wang \& Steinhardt 1998).

The redshift distribution of clusters in a survey that selects clusters according to some observable $O$ with redshift-dependent selection function $f(O, z)$ is given by

$$
\frac{d^{2} N(z)}{d z d \Omega}=\frac{r^{2}(z)}{H(z)} \int_{0}^{\infty} f(O, z) d O \int_{0}^{\infty} p(O \mid M, z) \frac{d n(z)}{d M} d M
$$

where $d n(z) / d M$ is the space density of dark halos in comoving coordinates, and $p(O \mid M, z)$ is the mass-observable relation, the probability that a halo of mass $M$ at redshift $z$ is observed as a cluster with observable property $O$. The utility of this probe hinges on the ability to robustly associate cluster observables such as X-ray luminosity or temperature, cluster galaxy richness, Sunyaev-Zel'dovich effect flux decrement, or weak lensing shear, with cluster mass (e.g., Borgani 2006).

The sensitivity of cluster counts to dark energy arises from two factors: geometry, the term multiplying the integral in Eq. (30) is the comoving volume element; and growth of structure, $d n(z) / d M$ depends on the evolution of density perturbations, cf. Eq. 15. The cluster mass function is also determined by the primordial spectrum of density perturbations; its near-exponential dependence upon mass is the root of the power of clusters to probe dark energy. 


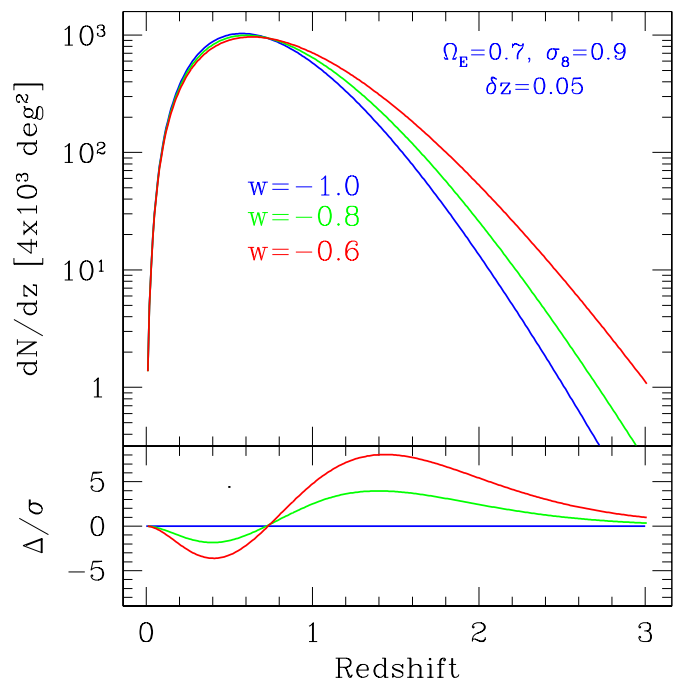

Figure 13: Predicted cluster counts for a survey covering 4,000 sq. deg. that is sensitive to halos more massive than $2 \times 10^{14} M_{\odot}$, for 3 flat cosmological models with fixed $\Omega_{\mathrm{M}}=0.3$ and $\sigma_{8}=0.9$. Lower panel shows differences between the models relative to the statistical errors. From Mohr (2005).

Fig. 13 shows the sensitivity to the dark energy equation-of-state parameter of the expected cluster counts for the South Pole Telescope and the Dark Energy Survey. At modest redshift, $z<0.6$, the differences are dominated by the volume element; at higher redshift, the counts are most sensitive to the growth rate of perturbations.

The primary systematic concerns are uncertainties in the mass-observable relation $p(O \mid M, z)$ and in the selection function $f(O, z)$. The strongest cosmological constraints arise for those cluster observables that are most strongly correlated with mass, i.e., for which $p(O \mid M, z)$ is narrow for fixed $M$, and which have a welldetermined selection function. There are several independent techniques both for detecting clusters and for estimating their masses using observable proxies. Future surveys will aim to combine two or more of these techniques to cross-check cluster mass estimates and thereby control systematic error. Measurement of the spatial correlations of clusters and of the shape of the mass function provide additional internal calibration of the mass-observable relation (Lima \& Hu 2004, Majumdar \& Mohr 2004).

With multi-band CCD imaging, clusters can be efficiently detected as enhancements in the surface density of early-type galaxies, and their observed colors provide photometric redshift estimates that substantially reduce the projection effects that plagued early optical cluster catalogs (Koester et al. 2007. Yee \& Gladders 2002). Weak lensing and dynamical studies show that cluster richness correlates with cluster mass (Johnston et al. 2007) and can be used to statistically calibrate mass-observable relations. Most of the cluster baryons reside in hot, X-ray emitting gas in approximate dynamical equilibrium in the dark matter potential well. Since X-ray luminosity is proportional to the square of the gas density, X-ray clusters are high-contrast objects, for which the selection func- 
tion is generally well-determined. Empirically, X-ray luminosity and temperature are both found to correlate more tightly than optical richness with virial mass (Arnaud 2005, Stanek et al. 2006).

The hot gas in clusters also Compton scatters CMB photons as they pass through, leading to the Sunyaev-Zel'dovich effect (SZE; Sunyaev \& Zeldovich 1970), a measurable distortion of the blackbody CMB spectrum. It can be detected for clusters out to high redshift (e.g., Carlstrom, Holder \& Reese 2002). Since the SZE flux decrement is linear in the gas density, it should be less sensitive to gas dynamics (Motl et al. 2005, Nagai 2006). Finally, weak gravitational lensing can be used both to detect and to infer the masses of clusters. Since lensing is sensitive to all mass along the line of sight, projection effects are the major concern for shear-selected cluster samples (Hennawi \& Spergel 2005: White, van Waerbeke \& Mackey 2002).

X-ray or SZE measurements also enable measurements of the baryonic gas mass in clusters; in combination with the virial mass estimates described above, this enables estimates of the baryon gas fraction, $f_{\text {gas }} \propto M_{\mathrm{B}} / M_{\text {tot }}$. The ratio inferred from X-ray/SZE measurements depends upon cosmological distance because the inferred baryon mass, $M_{\mathrm{B}} \propto d_{L}^{5 / 2}$ (X-ray) or $\propto d_{L}^{2}$ (SZE), and the inferred total mass from X-ray measurements $M_{\text {tot }} \propto d_{L}$. If clusters are representative sam-

ples of matter, then $f_{\text {gas }}(z) \propto d_{L}^{3 / 2}$ or 1 should be independent of redshift and $\approx \Omega_{\mathrm{B}} / \Omega_{\mathrm{M}}$; this will only be true for the correct cosmology (Allen et al. 2007, Rapetti \& Allen 2007).

\subsection{Baryon acoustic oscillations}

The peaks and troughs seen in the angular power spectrum of the CMB temperature anisotropy (see Fig. 5) arise from gravity-driven acoustic oscillations of the coupled photon-baryon fluid in the early Universe. The scale of these oscillations is set by the sound horizon at the epoch of recombination - the distance $s$ that sound waves in the fluid could have traveled by that time,

$$
s=\int_{0}^{t_{r e c}} c_{s}(1+z) d t=\int_{z_{r e c}}^{\infty} \frac{c_{s}}{H(z)} d z
$$

where the sound speed $c_{s}$ is determined by the ratio of the baryon and photon energy densities. The precise measurement of the angular scales of the acoustic peaks by WMAP has determined $s=147 \pm 2 \mathrm{Mpc}$. After recombination, the photons and baryons decouple, and the effective sound speed of the baryons plummets due to the loss of photon pressure; the sound waves remain imprinted in the baryon distribution and, through gravitational interactions, in the dark matter distribution as well. Since the sound horizon scale provides a "standard ruler" calibrated by the CMB anisotropy, measurement of the baryon acoustic oscillation (BAO) scale in the galaxy distribution provides a geometric probe of the expansion history.

In the galaxy power spectrum, this scale appears as a series of oscillations with amplitude of order 10\%, more subtle than the acoustic oscillations in the CMB because the impact of baryons on the far larger dark matter component is small. Measuring the BAO scale from galaxy clustering in the transverse and line-ofsight directions vields measurements of $r(z) / s$ and of $s H(z)$. respectively (Blake \& Glazebrook 2003, Hu \& Haiman 2003, Seo \& Eisenstein 2003). Spectroscopic 


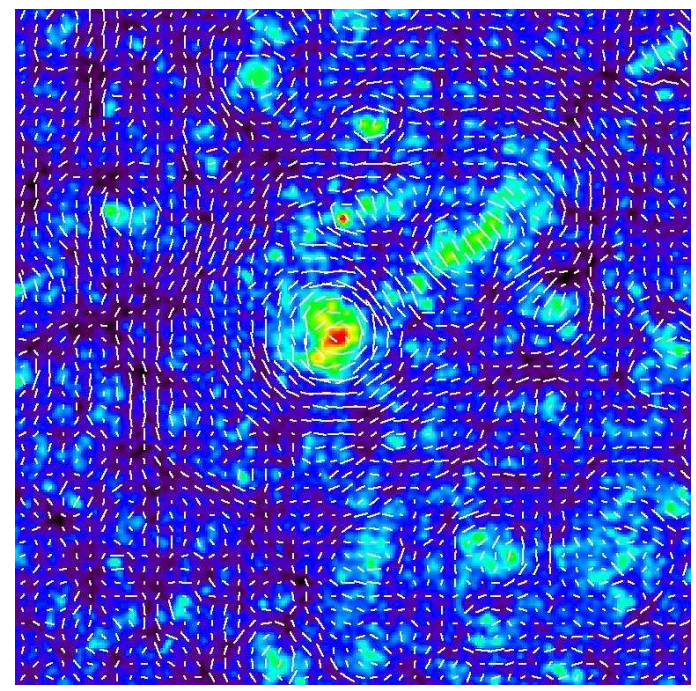

Figure 14: Cosmic shear field (white ticks) superimposed on the projected mass distribution from a cosmological N-body simulation: overdense regions are bright, underdense regions are dark. Note how the shear field is correlated with the foreground mass distribution. Figure courtesy of T. Hamana.

redshift surveys can probe both, while photometric surveys are mainly sensitive to transverse clustering. While determining these quantities with precision requires enormous survey volumes and millions of galaxies, N-body simulations suggest that the systematic uncertainties associated with BAO distance scale measurements are smaller than those of other observational probes of dark energy. Because such large numbers of galaxies are needed, BAO measurements provide distance estimates that are coarse-grained in redshift.

The main systematic uncertainties in the interpretation of BAO measurements are the effects of non-linear gravitational evolution, of scale-dependent differences between the clustering of galaxies and of dark matter (bias), and, for spectroscopic surveys, redshift distortions of the clustering, which can shift the BAO features. Numerical studies to date suggest that the resulting shift of the scale of the BAO peak in the galaxy power spectrum is at the percent level or less (Guzik, Bernstein \& Smith 2007, Seo \& Eisenstein 2007, Smith, Scoccimarro \& Sheth 2007), comparable to the forecast measurement uncertainty for future surveys but in principle predictable from high-resolution simulations.

\subsection{Weak gravitational lensing}

The gravitational bending of light by structures in the Universe distorts or shears the images of distant galaxies; see Fig. 14. This distortion allows the distribution of dark matter and its evolution with time to be measured, thereby probing the influence of dark energy on the growth of structure.

The statistical signal due to gravitational lensing by large-scale structure is termed "cosmic shear." The cosmic shear field at a point in the sky is estimated by locally averaging the shapes of large numbers of distant galaxies. The primary statistical measure of the cosmic shear is the shear angular power spectrum measured as a function of source-galaxy redshift $z_{s}$. (Additional information is 
obtained by measuring the correlations between shears at different redshifts or with foreground lensing galaxies.) The shear angular power spectrum is ( $\mathrm{Hu} \&$ Jain 2004, Kaiser 1992)

$$
P_{\ell}^{\gamma}\left(z_{s}\right)=\int_{0}^{z_{s}} d z \frac{H(z)}{d_{A}^{2}(z)}\left|W\left(z, z_{s}\right)\right|^{2} P_{\rho}\left(k=\frac{\ell}{d_{A}(z)} ; z\right),
$$

where $\ell$ denotes the angular multipole, the weight function $W\left(z, z_{s}\right)$ is the efficiency for lensing a population of source galaxies and is determined by the distance distributions of the source and lens galaxies, and $P_{\rho}(k, z)$ is the power spectrum of density perturbations.

As with clusters, the dark-energy sensitivity of the shear angular power spectrum comes from two factors: geometry - the Hubble parameter, the angulardiameter distance, and the weight functions; and growth of structure - through the evolution of the power spectrum of density perturbations. It is also possible to separate these effects and extract a purely geometric probe of dark energy from the redshift dependence of galaxy-shear correlations (Bernstein \& Jain 2004. Jain \& Taylor 2003). The three-point correlation of cosmic shear is also sensitive to dark energy (Takada \& Jain 2004).

The statistical uncertainty in measuring the shear power spectrum on large scales is (Kaiser 1992)

$$
\Delta P_{\ell}^{\gamma}=\sqrt{\frac{2}{(2 \ell+1) f_{\text {sky }}}}\left[P_{\ell}^{\gamma}+\frac{\sigma^{2}\left(\gamma_{i}\right)}{n_{\mathrm{eff}}}\right],
$$

where $f_{\text {sky }}$ is the fraction of sky area covered by the survey, $\sigma^{2}\left(\gamma_{i}\right)$ is the variance in a single component of the (two-component) shear, and $n_{\text {eff }}$ is the effective number density per steradian of galaxies with well-measured shapes. The first term in brackets, which dominates on large scales, comes from cosmic variance of the mass distribution, and the second, shot-noise term results from both the variance in galaxy ellipticities ("shape noise") and from shape-measurement errors due to noise in the images. Fig. 15 shows the dependence on the dark energy of the shear power spectrum and an indication of the statistical errors expected for a survey such as LSST, assuming a survey area of 15,000 sq. deg. and effective source galaxy density of $n_{\text {eff }}=30$ galaxies per sq. arcmin.

Systematic errors in weak lensing measurements arise from a number of sources (Huterer et al. 2006): incorrect shear estimates, uncertainties in galaxy photometric redshift estimates, intrinsic correlations of galaxy shapes, and theoretical uncertainties in the mass power spectrum on small scales. The dominant cause of galaxy shape measurement error in current lensing surveys is the anisotropy of the image point spread function (PSF) caused by optical and CCD distortions, tracking errors, wind shake, atmospheric refraction, etc. This error can be diagnosed since there are geometric constraints on the shear patterns that can be produced by lensing that are not respected by systematic effects. A second kind of shear measurement error arises from miscalibration of the relation between measured galaxy shape and inferred shear, arising from inaccurate correction for the circular blurring of galaxy images due to atmospheric seeing. Photometric redshift errors impact shear power spectrum estimates primarily through uncertainties in the scatter and bias of photometric redshift estimates in redshift bins (Huterer et al. 2006; Ma, Hu \& Huterer 2006). Any tendency of galaxies to align 


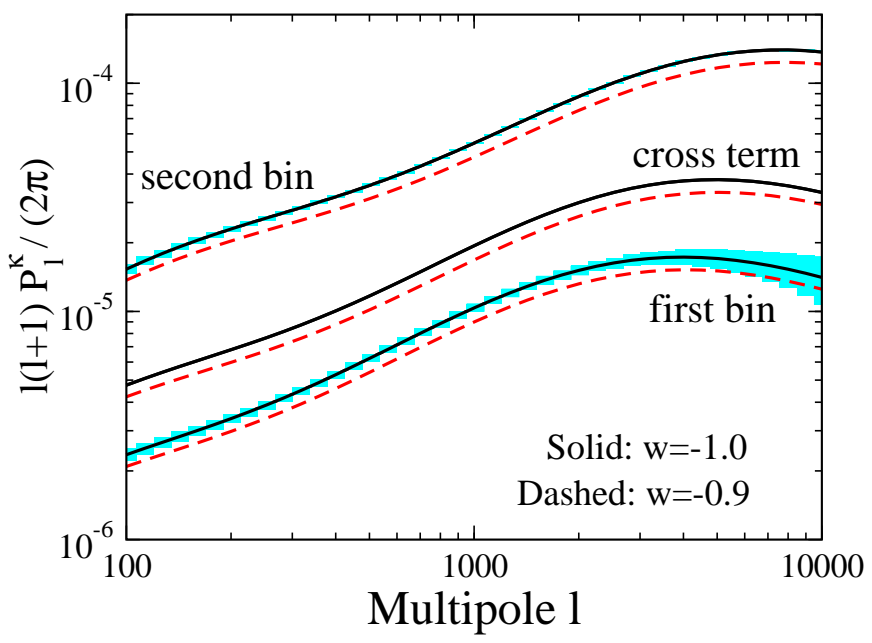

Figure 15: Cosmic shear angular power spectrum and statistical errors expected for LSST (see $\sqrt{8}$ ) for $w=-1$ and -0.9 . For illustration, results are shown for source galaxies in two broad redshift bins, $z_{s}=0-1$ (first bin) and $z_{s}=1-3$ (second bin); the cross-power spectrum between the two bins (cross term) is shown without the statistical errors.

with their neighbors - or to align with the local mass distribution — can be confused with alignments caused by gravitational lensing, thus biasing dark energy determinations (Heymans et al. 2006, Hirata \& Seljak 2004). Finally, uncertainties in the theoretical mass power spectrum on small scales could complicate attempts to use the high-multipole $(\ell \gtrsim$ several hundred) shear power spectrum to constrain dark energy. Fortunately, weak lensing surveys should be able to internally constrain the impact of such effects (Zentner, Rudd \& Hu 2007).

\subsection{Other probes}

While the four methods discussed above have the most probative power, a number of other methods have been proposed, offering the possibility of additional consistency checks. The Alcock-Paczynski test exploits the fact that the apparent shapes of intrinsically spherical cosmic structures depend on cosmology (Alcock \& Paczynski 1979). Since spatial clustering is statistically isotropic, the anisotropy of the two-point correlation function along and transverse to the line of sight has been proposed for this test. e.g.. using the Lvman-alpha forest (Hui, Stebbins \& Burles 1999).

Weak lensing of the CMB anisotropy by foreground clusters, in combination with lensing of galaxies, provides a potential geometric probe of dark energy (e.g., Hu, Holz \& Vale 2007).

The Integrated Sachs-Wolfe (ISW) effect provided a confirmation of cosmic acceleration, cf. §4.1.2. ISW impacts the large-angle structure of the CMB anisotropy, but low- $\ell$ multipoles are subject to large cosmic variance, limiting their power. Nevertheless, ISW is of interest because it may be able to show the imprint of large-scale dark-energy perturbations (Coble, Dodelson \& Frieman 1997; Hu \& Scranton 2004). 
Gravitational radiation from inspiraling binary neutron stars or black holes can serve as "standard sirens" to measure absolute distances. If their redshifts can be determined, then they could be used to probe dark energy through the Hubble diagram (Dalal et al. 2006).

Long-duration gamma-ray bursts have been proposed as standardizable candles (e.g., Schaefer 2003), but their utility as cosmological distance indicators that could be competitive with or complementary to SNe Ia has yet to be established (Friedman \& Bloom 2005). The angular size-redshift relation for double radio galaxies has also been used to derive cosmological constraints that are consistent with dark energy (Guerra, Daly \& Wan 2000). The optical depth for strong gravitational lensing (multiple imaging) of QSOs or radio sources has been proposed (Fukugita et al. 1992) and used (e.g., Chae 2007, Mitchell et al. 2005) to provide independent evidence for dark energy, though these measurements depend on modeling the density profiles of lens galaxies.

Polarization measurements from distant galaxy clusters in principle provide a sensitive probe of the growth function and hence dark energy (Cooray, Huterer \& Baumann 2004). The relative ages of galaxies at different redshifts, if they can be determined reliably, provide a measurement of $d z / d t$ and, from Eq. (13), measure the expansion history directly (Jimenez \& Loeb 2002). Measurements of the abundance of lensed arcs in galaxy clusters, if calibrated accurately, provide a probe of dark energy (Meneghetti et al. 2005).

As we have stressed, there is every reason to expect that at early times dark energy was but a tiny fraction of the energy density. Big bang nucleosynthesis and $\mathrm{CMB}$ anisotropy have been used to test this prejudice, and current data already indicate that dark energy at early times contributes no more than $\sim 5 \%$ of the total energy density (Bean. Hansen \& Melchiorri 2001; Doran \& Robbers 2006).

\subsection{Role of the CMB}

While the CMB provides precise cosmological constraints, by itself it has little power to probe dark energy (see Fig. 17). The reason is simple: the CMB provides a single snapshot of the Universe at a time when dark energy contributed but a tiny part of the total energy density (a part in $10^{9}$ for vacuum energy). Nonetheless, the CMB plays a critical supporting role by determining other cosmological parameters, such as the spatial curvature and matter density, to high precision, thereby considerably strengthening the power of the methods discussed above, cf. Fig. 8, It also provides the standard ruler for BAO measurements. Data from the Planck CMB mission, scheduled for launch in 2008, will complement those from dark energy surveys. If the Hubble parameter can be directly

measured to better than a few percent, in combination with Planck it would also provide powerful dark energy constraints ( $\mathrm{Hu}$ 2005).

\section{7 $\quad$ Probing new gravitational physics}

In $§ 5.2$ we discussed the possibility that cosmic acceleration could be explained by a modification of General Relativity on large scales. How can we distinguish this possibility from dark energy within GR and/or test the consistency of GR to explain cosmic acceleration? Since modified gravity can change both the Friedmann equation and the evolution of density perturbations, a strategy for testing 


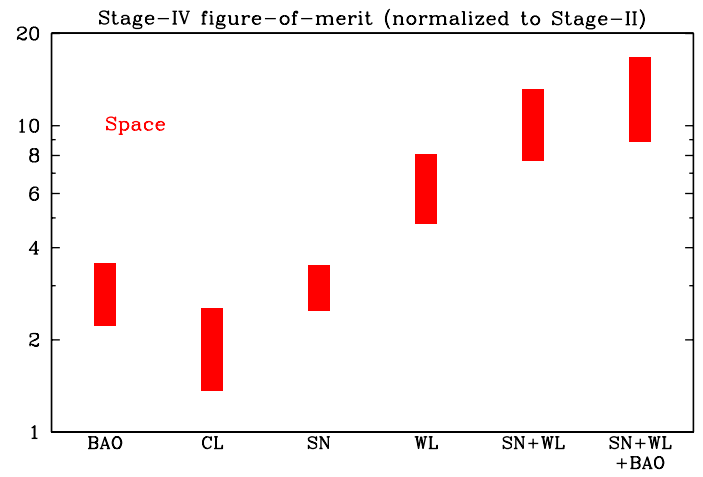

Figure 16: Relative statistical power of different dark energy space probes, separately and in combination, in constraining the DETF Figure of Merit (FoM) (see 111). Bars indicate estimated range of increase (allowing for uncertainties in systematic errors) in the FoM relative to present experiments. Adopted from the DETF report (Albrecht et al. 2006).

the consistency of GR and dark energy as the explanation for acceleration is to compare results from the geometric (expansion history) probes, e.g., SNe or $\mathrm{BAO}$, with those from the probes sensitive to the growth of structure, e.g., clusters or weak lensing. Differences between the two could be evidence for the need to modify GR (Knox, Song \& Tyson 2006). A first application of this idea to current data shows that standard GR passes a few modest consistency tests ( $\mathrm{Chu}$ \& Knox 2005, Wang et al. 2007).

Finally, any modification of gravity may have observable effects beyond cosmology, and precision solar system tests can provide important additional constraints (e.g., Lue, Scoccimarro \& Starkman 2004).

\subsection{Summary and comparison}

Four complementary cosmological techniques have the power to probe dark energy with high precision and thereby advance our understanding of cosmic acceleration: Weak Gravitational Lensing (WL); type Ia supernovae (SN); Baryon Acoustic Oscillations (BAO); and Galaxy Clusters (CL). To date, constraints upon the dark energy equation-of-state parameter have come from combining the results of two or more techniques, e.g., SN+BAO+CMB (see Fig. 8) or $\mathrm{BAO}+\mathrm{CMB}$ (see Table 1), in order to break cosmological parameter degeneracies. In the future, each of these methods, in combination with CMB information that constrains other cosmological parameters, will provide powerful individual constraints on dark energy; collectively, they should be able to approach percentlevel precision on $w$ at its best-constrained redshift, i.e., $w_{p}$ (see Fig. 17).

Table 2 summarizes these four dark energy probes, their strengths and weaknesses and primary systematic errors. Fig. 16 gives a visual impression of the statistical power of each of these techniques in constraining dark energy, showing how much each of them could be expected to improve our present knowledge of $w_{0}$ and $w_{a}$ in a dedicated space mission (Albrecht et al. 2006). 
Table 2: Comparison of dark energy probes.

\begin{tabular}{llll}
\hline \hline Method & Strengths & Weaknesses & Systematics \\
\hline WL & $\begin{array}{l}\text { growth+geometric, } \\
\text { statistical power }\end{array}$ & CDM assumption & $\begin{array}{l}\text { image quality, } \\
\text { photo-z }\end{array}$ \\
\hline SN & $\begin{array}{l}\text { purely geometric, } \\
\text { mature }\end{array}$ & $\begin{array}{l}\text { standard candle } \\
\text { assumption }\end{array}$ & $\begin{array}{l}\text { evolution, } \\
\text { dust }\end{array}$ \\
\hline BAO & largely geometric, & large samples & bias, \\
& low systematics & required & non-linearity \\
\hline CL & growth+geometric, & CDM assumption & $\begin{array}{l}\text { determining mass, } \\
\text { Selection function }\end{array}$ \\
& X-ray+SZ+optical & & selon \\
\hline \hline
\end{tabular}

\section{DARK ENERGY PROJECTS}

A diverse and ambitious set of projects to probe dark energy are in progress or being planned. Here we provide a brief overview of the observational landscape. With the exception of experiments at the LHC that might shed light on dark energy through discoveries about supersymmetry or dark matter, all planned experiments involve cosmological observations. Table 3 provides a representative sampling, not a comprehensive listing, of projects that are currently proposed or under construction and does not include experiments that have already reported results. All of these projects share the common feature of surveying wide areas to collect large samples of objects - galaxies, clusters, or supernovae.

The Dark Energy Task Force (DETF) report (Albrecht et al. 2006) classified dark energy surveys into an approximate sequence: on-going projects, either taking data or soon to be taking data, are Stage II; near-future, intermediate-scale projects are Stage III; and larger-scale, longer-term future projects are designated Stage IV. More advanced stages are in general expected to deliver tighter dark energy constraints, which the DETF quantified using the $w_{0}-w_{a}$ figure of merit (FoM) discussed in the Appendix (\$11.1). Stage III experiments are expected to deliver a factor $\sim 3-5$ improvement in the DETF FoM compared to the combined Stage II results, while Stage IV experiments should improve the FoM by roughly a factor of 10 compared to Stage II, though these estimates are only indicative and are subject to considerable uncertainties in systematic errors (see Fig. 16).

We divide our discussion into ground- and space-based surveys. Ground-based projects are typically less expensive than their space-based counterparts and can employ larger-aperture telescopes. The discovery of dark energy and many of the subsequent observations to date have been dominated by ground-based telescopes. On the other hand, HST (high-redshift SN observations), Chandra (X-ray clusters), and WMAP CMB observations have played critical roles in probing dark energy. While more challenging to execute, space-based surveys offer the advantages of observations unhindered by weather and by the scattering, absorption, and emission by the atmosphere, stable observing platforms free of time-changing gravitational loading, and the ability to continuously observe away from the sun and moon. They therefore have the potential for much improved control of systematic errors. 


\subsection{Ground-based surveys}

A number of projects are underway to detect clusters and probe dark energy using the SZE (see Sec. 7.2). These surveys are coordinated with optical surveys that can determine cluster redshifts. The Atacama Pathfinder EXperiment (APEX) survey in Chile will cover up to 1000 square degrees. The largest of these projects are the Atacama Cosmology Telescope (ACT) and the South Pole Telescope (SPT), the latter of which will carry out a 4,000 square degree survey.

A number of optical imaging surveys are planned or proposed which can study dark energy through weak lensing, clusters, and angular BAO using a single wide-area survey. These projects use telescopes of intermediate to large aperture and wide field-of-view, gigapixel-scale CCD cameras, and are deployed at the best astronomical sites in order to obtain deep galaxy photometry and shape measurements. They deliver photometric-redshift information through color measurements using multiple passbands. The ESO VLT Survey Telescope (VST) on Cerro Paranal will carry out public surveys, including the 1500 sq. deg. KIDS survey and a shallower, 5000 sq. deg. survey (ATLAS). The Panoramic Survey Telescope and Rapid Response System (Pan-STARRS)-1 uses a 1.8-m wide-field telescope to carry out several wide-area surveys from Haleakala; in the future, they hope to deploy $4 \times 1.8-\mathrm{m}$ telescopes at Mauna Kea in Pan-STARRS- 4 . The Dark Energy Survey (DES) will use a new 3 sq. deg. imager with red-sensitive CCDs on a 4-m telescope at Cerro Tololo Inter-American Observatory (CTIO) in Chile to carry out a 5,000 sq. deg. survey in 5 optical passbands, covering the same survey area as the SPT and partnering with the ESO VISTA Hemisphere Survey which will survey the same area in 3 near-infrared bands. Hyper SuprimeCam is a new wide-field imager planned for the Subaru telescope on Mauna Kea that will be used to carry out a deep survey over 2000 sq. deg. The Advanced Liquid-mirror Probe of Asteroids, Cosmology and Astrophysics (ALPACA) is a proposed rotating liquid mercury telescope that would repeatedly survey a long, narrow strip of the sky at CTIO. The most ambitious of these projects is the Large Synoptic Survey Telescope (LSST), which would deploy a multi-Gigapixel camera with 10 sq. deg. field-of-view on a new telescope on Cerro Pachon in Chile to survey $15,000 \mathrm{sq}$. deg. over 10 years.

Several large spectroscopic surveys have been designed to detect baryon acoustic oscillations by measuring $\sim 10^{5}-10^{9}$ galaxy and QSO redshifts using large multi-fiber spectrographs. WiggleZ is using the Anglo-Australian Telescope to collect spectra of 400,000 galaxies in the redshift range $0.5<z<1$. The Baryon Oscillation Sky Survey (BOSS) proposes to use the SDSS telescope in New Mexico to measure galaxy spectra out to $z=0.6$. The Hobby Eberly Telescope Dark energy EXperiment (HETDEX) plans to target Ly- $\alpha$ emitters at higher redshift, $2 \lesssim z \lesssim 4$. The Wide-Field Multi-Object Spectrograph (WFMOS), proposed for the Subaru telescope, would target galaxies at $z \lesssim 1.3$ and Lyman-break galaxies at $2.5 \lesssim z \lesssim 3.5$. The Physics of the Accelerating Universe (PAU) is a Spanish project to deploy a wide-field camera with a large number of narrow filters to measure coarse-grained galaxy spectra out to $z=0.9$.

Finally, the proposed Square Kilometer Array (SKA), an array of radio antennas with unprecedented collecting area, would probe dark energy using baryon acoustic oscillations and weak lensing of galaxies via measurements of the $21-\mathrm{cm}$ line signature of neutral hydrogen (HI). The Hubble Sphere Hydrogen Survey (HSHS) aims to carry out a 21-cm BAO survey on a shorter timescale. 
Table 3: Dark energy projects proposed or under construction. Stage refers to the DETF time-scale classification.

\begin{tabular}{|c|c|c|c|}
\hline Survey & Description & Probes & Stage \\
\hline \multicolumn{4}{|l|}{ Ground-based: } \\
\hline $\mathrm{ACT}$ & SZE, 6-m & CL & II \\
\hline APEX & $\mathrm{SZE}, 12-\mathrm{m}$ & $\mathrm{CL}$ & II \\
\hline SPT & SZE, $10-\mathrm{m}$ & CL & II \\
\hline VST & Optical imaging, $2.6-\mathrm{m}$ & $\mathrm{BAO}, \mathrm{CL}, \mathrm{WL}$ & II \\
\hline Pan-STARRS 1(4) & Optical imaging, $1.8-\mathrm{m}(\times 4)$ & All & $\mathrm{II}(\mathrm{III})$ \\
\hline DES & Optical imaging, 4-m & All & III \\
\hline Hyper Suprime-Cam & Optical imaging, 8-m & WL,CL,BAO & III \\
\hline ALPACA & Optical imaging, 8-m & $\mathrm{SN}, \mathrm{BAO}, \mathrm{CL}$ & III \\
\hline LSST & Optical imaging, $6.8-\mathrm{m}$ & All & IV \\
\hline AAT WiggleZ & Spectroscopy, 4-m & $\mathrm{BAO}$ & II \\
\hline HETDEX & Spectroscopy, 9.2-m & $\mathrm{BAO}$ & III \\
\hline PAU & Multi-filter imaging, 2-3-m & $\mathrm{BAO}$ & III \\
\hline SDSS BOSS & Spectroscopy, 2.5-m & $\mathrm{BAO}$ & III \\
\hline WFMOS & Spectroscopy, 8-m & $\mathrm{BAO}$ & III \\
\hline HSHS & $21-\mathrm{cm}$ radio telescope & $\mathrm{BAO}$ & III \\
\hline SKA & $\mathrm{km}^{2}$ radio telescope & $\mathrm{BAO}, \mathrm{WL}$ & IV \\
\hline \multicolumn{4}{|l|}{ Space-based: } \\
\hline ADEPT & Spectroscopy & $\mathrm{BAO}, \mathrm{SN}$ & IV \\
\hline DESTINY & Grism spectrophotometry & $\mathrm{SN}$ & IV \\
\hline SNAP & Optical+NIR+spectro & All & IV \\
\hline \multicolumn{4}{|c|}{ Proposed ESA Missions } \\
\hline DUNE & Optical imaging & $\mathrm{WL}, \mathrm{BAO}, \mathrm{CL}$ & \\
\hline SPACE & Spectroscopy & $\mathrm{BAO}$ & \\
\hline eROSITA & X-ray & $\mathrm{CL}$ & \\
\hline \multicolumn{4}{|l|}{ CMB Space Probe } \\
\hline Planck & SZE & CL & \\
\hline \multicolumn{4}{|l|}{ Beyond Einstein Probe } \\
\hline Constellation-X & X-ray & CL & IV \\
\hline
\end{tabular}

\subsection{Space-based surveys}

Three of the proposed space projects are candidates for the Joint Dark Energy Mission (JDEM), a joint mission of the U.S. Department of Energy (DOE) and the NASA Beyond Einstein program, targeted at dark energy science. SuperNova/Acceleration Probe (SNAP) proposes to study dark energy using a dedicated 2-m class telescope. With imaging in 9 optical and near-infrared passbands and follow-up spectroscopy of supernovae, it is principally designed to probe $\mathrm{SNe}$ Ia and weak lensing, taking advantage of the excellent optical image quality and near-infrared transparency of a space-based platform. Fig. 17 gives an illustration of the statistical constraints that the proposed SNAP mission could achieve, by combining $\mathrm{SN}$ and weak lensing observations with results from the Planck $\mathrm{CMB}$ mission. This forecast makes use of the Fisher information matrix de- 

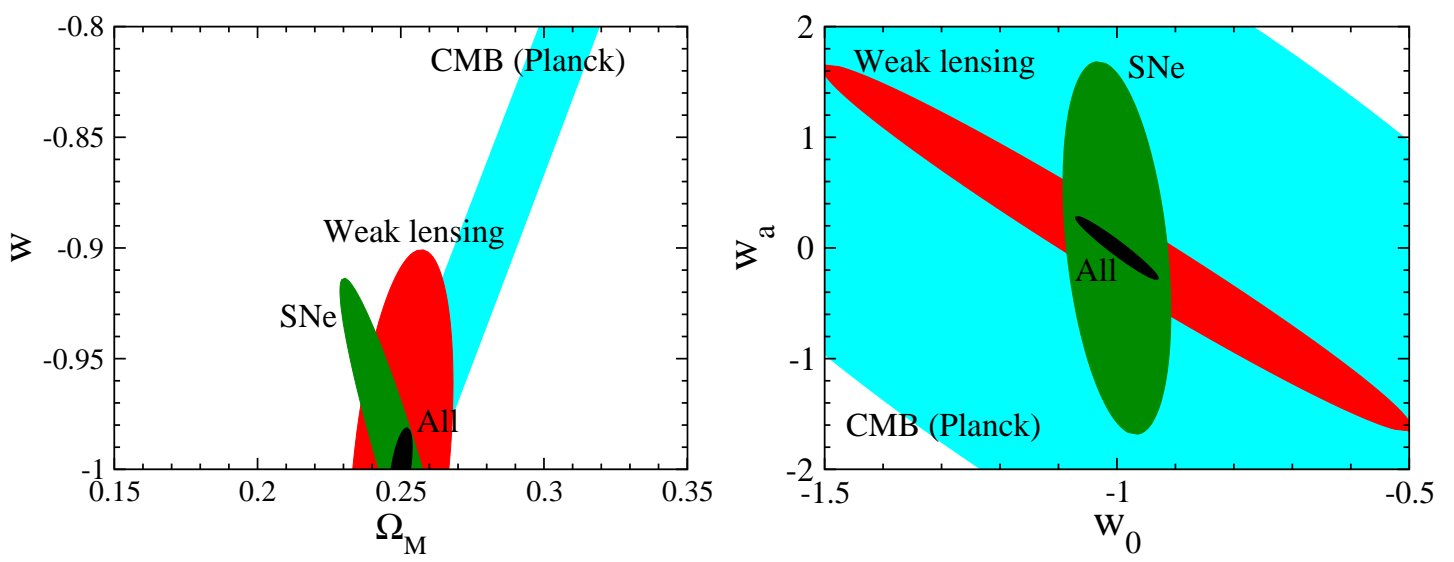

Figure 17: Illustration of forecast constraints on dark energy parameters. Shown are $68 \%$ C.L. uncertainties for one version of the proposed SNAP experiment, which combines a narrow-area survey of 2000 SNe to $z=1.7$ and a weak lensing survey of $1000 \mathrm{sq}$. deg. Left panel: Constraints in the $\Omega_{\mathrm{M}}-w$ plane, assuming constant $w$; the vertical axis can also be interpreted as the pivot value $w_{p}$ for a time-varying equation of state. Right panel: Constraints in the $w_{0}-w_{a}$ plane for time-varying dark energy equation of state, marginalized over $\Omega_{\mathrm{M}}$ for a flat Universe.

scribed in the Appendix (\$11.2). The Dark Energy Space Telescope (DESTINY) would use a similar-size telescope with a near-infrared grism spectrograph to study supernovae. The Advanced Dark Energy Physics Telescope (ADEPT) is a spectroscopic mission with the primary goal of constraining dark energy via baryon acoustic oscillations at $z \sim 2$ as well as supernovae. Another proposed mission within the NASA Beyond Einstein program is Constellation-X, which could observe X-ray clusters with unprecedented sensitivity.

There is one European Space Agency (ESA) mission nearing launch and two concepts under study. The Planck mission, planned for launch in late 2008, in addition to pinning down other cosmological parameters important for dark energy, will detect thousands of galaxy clusters using the SZE. Dark Universe Explorer (DUNE) and SPACE are optical missions to study dark energy using weak lensing and baryon acoustic oscillations, respectively. Finally, the extended ROentgen Survey with an Imaging Telescope Array (eROSITA), a German-Russian collaboration, is a planned X-ray telescope that will study dark energy using the abundance of X-ray clusters.

\section{DARK ENERGY \& COSMIC DESTINY}

One of the first things one learns in cosmology is that geometry is destiny: a closed (positively curved) Universe eventually recollapses, and an open (flat or negatively curved) Universe expands forever. Provided the Universe contains only matter and $\Lambda=0$, this follows directly from Eq. (2). The presence of dark energy severs this well known connection between geometry and destiny and raises fundamental issues involving the distant future of our Universe Krauss \& Turner 1995).

To illustrate the geometry-destiny connection, we can rewrite Eq. (2) in terms 


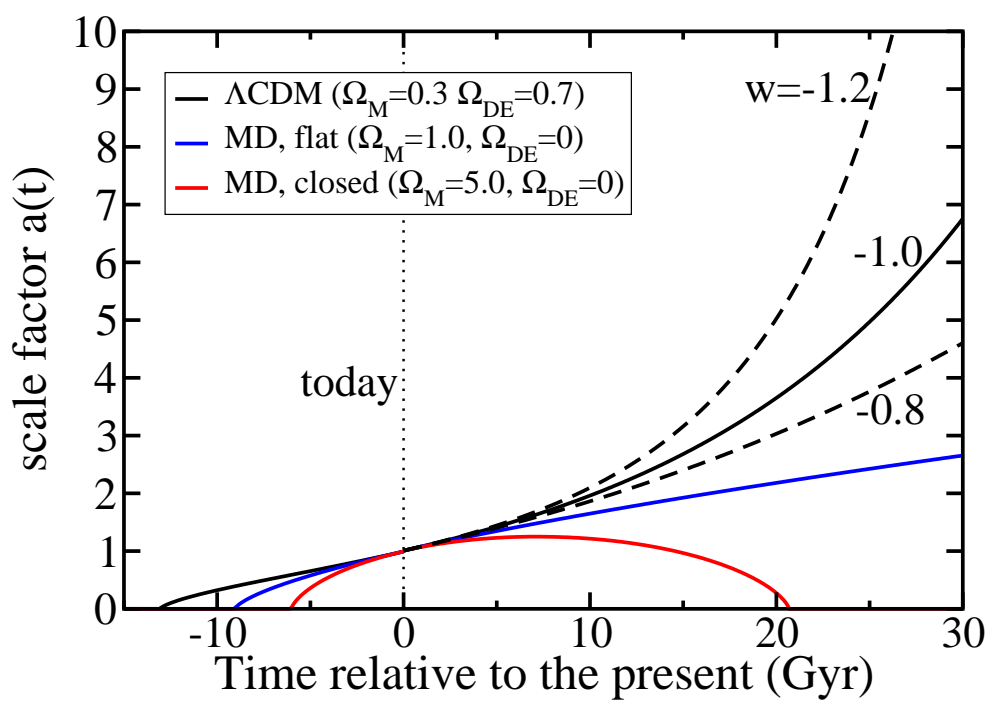

Figure 18: Evolution of the FRW scale factor in models with and without dark energy. Upper four curves are for flat models. Dashed curves denote models with $w=-0.8$ or -1.2 and $\Omega_{\mathrm{M}}=0.3$. MD denotes matter-dominated models.

of an effective potential and a kinetic-energy term,

$$
V_{\text {eff }}(a)+\dot{a}^{2}=0 \quad V_{\text {eff }}(a)=k-\Omega_{0} H_{0}^{2} a^{-\left(1+3 w_{\mathrm{T}}\right)},
$$

where $w_{\mathrm{T}}$ is the ratio of the total pressure to the total energy density (including all components). If $w_{\mathrm{T}}>-1 / 3$, as would be the case with only matter and radiation, then the second term in $V_{\text {eff }}$ increases monotonically from $-\infty$ to 0 as $a$ goes from 0 to $\infty$, which means that $V_{\text {eff }}$ rises from $-\infty$ to $k$. For $k>0$, there is a value of $a$ where $V_{\text {eff }} \rightarrow 0$, at which point $\dot{a}$ must go to zero and $a$ achieves its maximum value. For $k=0, \dot{a}$ only vanishes for $a \rightarrow \infty$; and for $k<0, \dot{a}$ remains positive even as $a \rightarrow \infty$.

With dark energy there is a new twist: since the dark energy density decreases more slowly than that of matter or radiation, as the Universe expands dark energy eventually dominates the second term in $V_{\text {eff }}$. Thereafter, $V_{\text {eff }}$ decreases monotonically, since $w_{\mathrm{T}} \simeq w_{\mathrm{DE}}<-1 / 3$, approaching $-\infty$ as $a \rightarrow \infty$. Provided that $\rho_{\mathrm{DE}}>0$ and that $w_{\mathrm{DE}}$ remains negative, if the scale factor becomes large enough for dark energy to dominate, which happens unless $\Omega_{\mathrm{M}}>1 \gg \Omega_{\mathrm{DE}}$, then the Universe will expand forever, irrespective of $k$.

If dark energy is vacuum energy, acceleration will continue, and the expansion will become exponential, leading to a "red out" of the Universe. To see this, consider the comoving distance to fixed redshift $z$ at time $t$ during the epoch of exponential expansion:

$$
r(z, t)=\int_{a(t) /(1+z)}^{a(t)} \frac{d a}{a^{2} H} \simeq z H_{0}^{-1} \exp \left[-H_{0}\left(t-t_{0}\right)\right] .
$$

The exponential decrease of this distance implies that the number of galaxies below a fixed redshift shrinks exponentially. By contrast, in the Einstein-de 
Sitter model with $\Omega_{\mathrm{M}}=1$, this distance increases as $t^{1 / 3}$, so that the number of galaxies with redshift less than a fixed value grows slowly. Alternatively, Eq. 35 implies that the redshift for a galaxy at current distance $r$ grows exponentially. Galaxies beyond the Local Group, $r \gtrsim 1-2 \mathrm{Mpc}$, will be redshifted beyond detectability on a timescale of $t-t_{0} \sim 100 \mathrm{Gyr}$ (e.g., Busha et al. 2003). The Milky Way will remain gravitationally bound to the Local Group, which will appear as a static, "island Universe." Even the CMB, the other key evidence of a once-hot, expanding Universe, will be redshifted to undetectabilitv (Krauss \& Scherrer 2007).

If dark energy is a scalar field, then eventually the field relaxes to the minimum of its potential; see Fig 10. If the minimum of the potential energy is precisely zero, the Universe will again become matter dominated and return to decelerated expansion, restoring the link between geometry and destiny. If the minimum of the scalar field potential has negative energy density, the energy of dark matter and of scalar field energy will eventually cancel, leading to recollapse, irrespective of $k$. If the potential energy at the minimum is positive and larger than a critical value that depends on $\Omega_{\mathrm{M}}$ (the critical value is zero for $\Omega_{\mathrm{M}} \leq 1$ and small for $\Omega_{\mathrm{M}}>1$ ), then accelerated expansion will eventually ensue again and as discussed above, the Universe will experience a "red-out." These possibilities are illustrated in Fig. 18.

Finally, the possibility of $w_{\mathrm{DE}}<-1$ deserves special comment. In this case, the energy density of dark energy actually increases with time, $\rho_{D E} \propto a^{\beta}$, where $\beta \equiv-3(1+w)>0$. In turn, the scale factor grows very rapidly and reaches infinite size in a finite time:

$$
a(t) \simeq \frac{1}{\left[1-\beta H_{0}\left(t-t_{0}\right) / 2\right]^{2 / \beta}}, \quad\left(t_{\infty}-t_{0}\right) \simeq \frac{2}{\beta H_{0}} .
$$

This is the "big rip" scenario of Caldwell, Kamionkowski \& Weinberg (2003). Once the density of dark energy exceeds that of any structure - from clusters to atoms - that structure is torn apart.

The presence of dark energy severs the simple relation between geometry and destiny, links destiny to an understanding of dark energy, raises the specter of a bleak future for cosmologists, and raises a deep question (Krauss \& Turner 1995): can we ever determine the future of the Universe with certainty? As a thought experiment, ignore the current epoch of accelerated expansion and imagine instead that the Universe has been determined to be matter dominated and that it is flat. We might be tempted to conclude that the Universe will expand forever at an ever-decreasing rate. However, no matter how precise our measurements are, there could be a small cosmological constant lurking below the threshold of detectability. For example, if the vacuum energy density were one billionth of the present matter density, after a factor of 1000 in expansion vacuum energy would come to dominate. If it were positive, exponential expansion would eventually ensue; if negative, the Universe would ultimately recollapse. Only a fundamental understanding of the constituents of the Universe and their relative abundances could deliver certainty about the destiny of the Universe.

\section{CONCLUDING REMARKS}

Ten years after its discovery, the acceleration of the expansion of the Universe is now firmly established. The physical origin of this phenomenon, however, remains 
a deep mystery, linked to other important problems in physics and astronomy. At present, the simplest explanation, vacuum energy, is consistent with all extant data, but theory provides no understanding of why it should have the requisite small value. Probing the history of cosmic expansion with much greater precision (few percent vs. current 10\%) offers the best hope of pointing us down the correct path to a solution. An impressive array of experiments with that aim are underway or planned, and we believe that significant progress will be made in the next fifteen years.

We conclude with our list of the ten important take-home facts about cosmic acceleration and dark energy, followed by our views on the key open issues and challenges for the future.

\subsection{Take-home facts}

10.1.1 Strong Evidence For accelerated Expansion. Since the SN discovery of acceleration, several hundred supernovae have been observed over a broader range of redshifts, substantially strengthening the case both statistically and by reducing sources of systematic error. Further, independent of GR and based solely upon the SN Hubble diagram, there is very strong $(5 \sigma)$ evidence that the expansion of the Universe accelerated recently (Shapiro \& Turner 2006).

10.1.2 DARK ENERGY AS THE CAUSE OF COSMIC ACCELERATION. Within GR, accelerated expansion cannot be explained by any known form of matter or energy but can be accommodated by a nearly smooth form of energy with large negative pressure, known as dark energy, that accounts for about $75 \%$ of the Universe.

10.1.3 INDEPENDENT EVIDENCE FOR DARK ENERGY. In the context of the cold dark matter model of structure formation, CMB and large-scale structure data provide independent evidence that the Universe contains a smooth form of energy which accounts for about $75 \%$ of the total and which only came to dominate after essentially all of the observed structure had formed. Thus, structure formation independently points to a negative-pressure (with $w \lesssim-1 / 3$ ), dark energy accounting for the bulk of the Universe.

10.1.4 VACUUM ENERGY AS DARK ENERGY. The simplest explanation for dark energy is the energy associated with the vacuum; it is mathematically equivalent to a cosmological constant. However, all attempts to compute the vacuum energy density from the zero-point energies of all quantum fields yield a result that is many orders of magnitude too large or infinite.

10.1.5 Current observational status. Taken together, all the current data provide strong evidence for the existence of dark energy; they constrain the fraction of critical density contributed by dark energy, $0.76 \pm 0.02$, and the equation-of-state parameter, $w \approx-1 \pm 0.1$ (stat) \pm 0.1 (sys), assuming that $w$ is constant. This implies that the Universe began accelerating at redshift $z \sim 0.4$ and age $t \sim 10$ Gyr. These results are robust - data from any one method can be removed without compromising the constraints - and they are not substantially weakened by dropping the assumption of spatial flatness. Relaxing the assumption that $w$ is constant and parametrizing its variation as $w(z)=w_{0}+w_{a}(1-a)$, the current observational constraints are considerably weaker, $\Omega_{\mathrm{DE}} \approx 0.7 \pm 0.15$, $w_{0} \approx-1 \pm 0.2, w_{a} \approx 0 \pm 1$, and provide no evidence for variation of $w$. 
10.1.6 DARK THEORY: DARK ENERGY OR NEW GRAVITATIONAL PHYSICS? There is no compelling model for dark energy. Beyond vacuum energy, there are many intriguing ideas, including a new light scalar field and the influence of additional spatial dimensions. In many of these models, time-varying dark energy is expected. On the other hand, cosmic acceleration could be a manifestation of gravitational physics beyond GR rather than dark energy. While interesting, there is as yet no self-consistent model for the new gravitational physics that is also consistent with the large body of data that constrains theories of gravity.

10.1.7 Dark Destiny. The destiny of the Universe depends crucially upon the nature of the dark energy. All three fates - recollapse or continued expansion with and without slowing - are possible. The existence of dark energy raises the issue of cosmic uncertainty: can we determine the mass/energy content with sufficient precision to rule out the possibility that a tiny dark energy component today may dominate in the distant future?

10.1.8 At THE NEXUS OF MANY MYSTERIES. Because of its multiple close connections to important problems in both physics and astronomy, cosmic acceleration may be the most profound mystery in science. Its solution could shed light on or be central to unraveling other important puzzles, including the cause of cosmic inflation, the vacuum-energy problem, supersymmetry and superstrings, neutrino mass, new gravitational physics, and even dark matter.

10.1.9 The Two BIG Questions. Today, the two most pressing questions about cosmic acceleration are: Is dark energy something other than vacuum energy? Does GR self-consistently describe cosmic acceleration? Establishing that $w \neq-1$ or that it varies with time, or that dark energy clusters, would rule out vacuum energy. Establishing that the values of $w$ determined by the geometric and growth of structure methods are not equal could point toward a modification of gravity as the cause of accelerated expansion.

10.1.10 Probing DARK ENERGY. An impressive array of space- and groundbased observations, using SNe, weak lensing, clusters, and baryon acoustic oscillations, are in progress or are being planned. They should determine $w_{p}$, the equation-of-state parameter at the redshift where it is best determined, at the percent level and its time variation $w_{a}$ at the $10 \%$ level, dramatically improving our ability to discriminate between vacuum energy and something more exotic as well as testing the self-consistency of GR to explain cosmic acceleration. Laboratory and accelerator-based experiments could also shed light on dark energy.

\subsection{Open issues and challenges}

10.2.1 Clustering OF DARK ENERGy. While vacuum energy is uniform, dynamical forms of dark energy can be inhomogeneous, making dark energy clustering a potential additional probe of dark energy. However, since dark energy is likely to cluster only weakly and on the largest scales, the prospects for clustering as a probe of dark energy are not high. Nonetheless, discovering that dark energy clusters would rule out vacuum energy. Current constraints on the clustering of dark energy are weak, and there may be better ideas about measuring dark-energy clustering.

10.2.2 DARK ENERGY AND MATTER. In scalar field models of dark energy, there is a new, very light $\left(m \lesssim H_{0} \sim 10^{-33} \mathrm{eV}\right)$ scalar particle which can couple to matter and thereby give rise to new long-range forces with potentially 
observable consequences. Such an interaction could perhaps help explain the near-coincidence between the present densities of dark matter and dark energy or change the dynamics of dark matter particles, though it is constrained by astrophysical and cosmological observations to be of at most gravitational strength (Carroll 1998, Gradwohl \& Frieman 1992). A coupling to ordinary matter would have even larger observable effects and is highly constrained.

10.2.3 DESCRIBING COSMIC ACCELERATION AND DARK ENERGY. In the absence of theoretical guidance, the equation-of-state parameter $w \equiv p / \rho$ is a convenient way of characterizing dark energy and its effects on the expansion. One can instead take a more agnostic approach and interpret results in terms of the kinematics of the expansion or the energy density. Further, it is worth exploring improved descriptions of dark energy that both yield physical insight and are better matched to the observations.

10.2.4 Systematic ERrors. All of the techniques used to probe dark energy are limited by systematic errors. The sources of systematic error include: luminosity evolution and dust extinction uncertainties (for SNe Ia); shape measurement systematics, photometric redshift errors, and theoretical modeling of the matter power spectrum (for weak lensing); galaxy biasing, non-linearity, and redshift distortions (for $\mathrm{BAO}$ ); and the uncertain relations between cluster mass and its observable proxies (for galaxy clusters). Improvements in all of these will be critical to realizing the full potential of planned observations to probe dark energy and will have beneficial effects more broadly in astronomy.

10.2.5 DARK Energy Theory. The grandest challenge of all is a deeper understanding of the cause of cosmic acceleration. What is called for is not the invention of ad hoc models based upon clever ideas or new potentials, but rather a small number of theoretical models that are well motivated by fundamental physics and that make specific enough predictions to be falsified.

10.2.6 How MUCH Is ENOUGH? Given its profound implications and the absence of a compelling theory, dark energy is the exemplar of high-risk, highgain science. Carrying out the most ambitious proposed dark energy projects - JDEM and LSST - to attain percent level precision will cost more than one billion dollars. While they will yield much tighter parameter constraints, there is no guarantee that they will deliver deeper understanding of dark energy. If they are able to exclude vacuum energy or demonstrate the inconsistency of GR, the implications would be revolutionary. On the other hand, if they yield results consistent with vacuum energy, it would constitute an important test of the "null hypothesis" and provide a set of cosmological parameters that will satisfy the needs for astrophysical cosmology for the foreseeable future. In this case, unless there are new theoretical developments pointing to different or more decisive probes of compelling dark energy theories, there is likely to be little enthusiasm for continuing on to even more expensive dark energy projects.

There is no doubt that pursuing the origin of cosmic acceleration will continue to be a great intellectual adventure for the next fifteen years. Even if these ambitious projects do not solve this riddle, they will at least sharpen the problem and will certainly produce a wealth of survey data that will benefit many areas of astronomy for decades to come. 


\section{Acknowledgements}

We thank Andy Albrecht, Gary Bernstein, Roger Blandford, Ed Copeland, Wendy Freedman, Don Goldsmith, Wayne Hu, Rocky Kolb, Eric Linder, Adam Riess, Martin White, and Bruce Winstein for helpful comments on an earlier draft of this article. We also acknowledge the Aspen Center for Physics, where part of this review was written. This work was supported in part by the DOE at Fermilab and at the University of Chicago and by the KICP NSF Physics Frontier Center grant PHY-0114422.

\section{APPENDIX}

\subsection{Figure(s) of merit}

How do we compare the dark energy "reach" of different methods and different experiments? We cannot quantify the probative power of dark energy methods in a strictly model-independent way, since we do not know which aspects of the expansion history are most important to measure. Nevertheless, some useful figures of merit (FoMs) have been proposed to facilitate comparison of methods and experimental designs. Examples include the volume of the uncertainty ellipsoid for the dark energy parameters or the thickness of the ellipsoid in its narrowest direction (Huterer \& Turner 2001). In the Fisher matrix approach (\$11.2), these correspond to the inverse square root of the determinant and the largest eigenvalue of the Fisher matrix, respectively. A special case of the volume FoM is the inverse area of the Fisher-matrix-projected ellipse in the $w_{0}-w_{a}$ plane,

$$
\mathrm{FoM} \propto\left[\sigma\left(w_{0}\right) \sigma\left(w_{a}\right)\right]^{-1} \propto\left(\operatorname{det} F^{w_{0} w_{a}}\right)^{1 / 2},
$$

where $F^{w_{0} w_{a}}$ is the Fisher matrix projected onto the $w_{0}-w_{a}$ plane. This choice was adopted by the Dark Energy Task Force as a metric for comparing methods and surveys and is shown in relative terms for Stage IV space-based experiments in Fig. 16. The DETF FoM provides a simple yet useful metric for comparison, as it takes into account the power of experiments to measure the temporal variation of $w$. For generalizations, see Albrecht \& Bernstein (2007).

\subsection{Fisher information matrix}

The Fisher information matrix formalism allows a quick and easy way to estimate errors on cosmological parameters, given errors in observable quantities, and is particularly useful in experimental design. The Fisher matrix is defined as the (negative) Hessian of the $\log$-likelihood function $\mathcal{L}$,

$$
F_{i j} \equiv\left\langle-\frac{\partial^{2} \ln \mathcal{L}}{\partial p_{i} \partial p_{j}}\right\rangle=\mu_{, i}^{T} \mathbf{C}^{-1} \mu_{, j}+\frac{1}{2} \operatorname{Tr}\left[\mathbf{C}^{-1} \mathbf{C}_{, i} \mathbf{C}^{-1} \mathbf{C}_{, j}\right] .
$$

The second equality follows by assuming that $\mathcal{L}$ is Gaussian in the observables; here $\mu$ is the vector of mean values of the observables, $\mathbf{C}$ is their covariance matrix, and ${ }_{, i}$ denotes a derivative with respect to $i$ th model parameter $p_{i}$. The parameter vector $\vec{p}$ includes both cosmological and any other model parameters needed to characterize the observations. This expression often simplifies - for example, for $N$ observable quantities with mean values $\mathbf{O}_{\alpha}$ and a covariance matrix $\mathbf{C}$ that does not depend on the cosmological parameters, the Fisher matrix becomes $F_{i j}=\sum_{\alpha, \beta}\left(\partial \mathbf{O}_{\alpha} / \partial p_{i}\right) \mathbf{C}_{\alpha \beta}^{-1}\left(\partial \mathbf{O}_{\beta} / \partial p_{j}\right)$. 
By the Cramer-Rao inequality, a model parameter $p_{i}$ cannot be measured to a precision better than $1 / \sqrt{F_{i i}}$ when all other parameters are fixed, or a precision $\sqrt{F_{i i}^{-1}}$ when all other parameters are marginalized over. In practice, the Fisher matrix is a good approximation to the uncertainties as long as the likelihood can be approximated by a Gaussian, which is generally the case near the peak of the likelihood and therefore in cases when the parameters are measured with small errors. Conversely, if the errors are large, then the likelihood is typically nonGaussian, and the constraint region is no longer elliptical but characteristically

banana-shaped, as in Fig. 8. In this case, the Fisher matrix typically underestimates the true parameter errors and degeneracies, and one should employ a Monte Carlo approach to error estimation.

\section{References}

Afshordi N, Loh YS, Strauss MA. 2004. Phys. Rev. D69:083524

Aguirre AN. 1999. Astrophys. J. Letters 512:L19-L22

Albrecht A, Bernstein G. 2007. Phys. Rev. D75:103003

Albrecht A, Bernstein G, Cahn R, Freedman WL, Hewitt J, et al. 2006. astroph/0609591

Alcock C, Paczynski B. 1979. Nature 281:358

Allen SW, Rapetti DA, Schmidt RW, Ebeling H, Morris G, Fabian AC. 2007. astro-ph/0706.0033

Allen SW, Schmidt RW, Ebeling H, Fabian AC, van Speybroeck L. 2004. Mon. Not. Roy. Astron. Soc. 353:457

Alnes H, Amarzguioui M, Gron O. 2006. Phys. Rev. D73:083519

Armendariz-Picon C, Mukhanov VF, Steinhardt PJ. 2000. Phys. Rev. Lett. 85:4438-4441

Arnaud M. 2005. In Background Microwave Radiation and Intracluster Cosmology, eds. F Melchiorri, Y Rephaeli

Arnett WD. 1982. Astrophys. J. 253:785-797

Astier P, et al. 2006. Astron. ES Astrophys. 447:31-48

Bacon DJ, Refregier AR, Ellis RS. 2000. MNRAS 318:625-640

Bean R, Hansen SH, Melchiorri A. 2001. Phys. Rev. D64:103508

Bernstein GM, Jain B. 2004. Astrophys. J. 600:17-25

Blake C, Glazebrook K. 2003. Astrophys. J. 594:665-673

Bondi H, Gold T. 1948. MNRAS 108:252

Borgani S. 2006. astro-ph/0605575

Boughn S, Crittenden R. 2004. Nature 427:45-47

Bousso R, Polchinski J. 2000. JHEP 06:006

Busha MT, Adams FC, Wechsler RH, Evrard AE. 2003. Astrophys. J. 596:713724

Caldwell RR. 2002. Phys. Lett. B545:23-29

Caldwell RR, Kamionkowski M, Weinberg NN. 2003. Phys. Rev. Lett. 91:071301 
Caldwell RR, Linder EV. 2005. Phys. Rev. Lett. 95:141301

Carlstrom JE, Holder GP, Reese ED. 2002. Ann. Rev. Astron. Astrophys. 40:643-680

Carroll SM. 1998. Phys. Rev. Lett. 81:3067-3070

Carroll SM. 2001. Living Rev. Rel. 4:1

Carroll SM, Duvvuri V, Trodden M, Turner MS. 2004. Phys. Rev. D70:043528

Carroll SM, Hoffman M, Trodden M. 2003. Phys. Rev. D 68:023509

Carroll SM, Press WH, Turner EL. 1992. Ann. Rev. Astron. Astrophys. 30:499542

Chae KH. 2007. Astrophys. J. Letters 658:L71-L74

Chu M, Knox L. 2005. Astrophys. J. 620:1-6

Coble K, Dodelson S, Frieman JA. 1997. Phys. Rev. D55:1851-1859

Cooray A, Huterer D, Baumann D. 2004. Phys. Rev. D69:027301

Copeland EJ, Sami M, Tsujikawa S. 2006. Int. J. Mod. Phys. D15:1753-1936

Corasaniti PS, Copeland EJ. 2003. Phys. Rev. D67:063521

Dalal N, Holz DE, Hughes SA, Jain B. 2006. Phys. Rev. D 74:063006

de Sitter W. 1917. Proc. Acad. Sci. 19:1217

Deffayet C. 2001. Phys. Lett. B502:199-208

Dodelson S. 2003. Modern cosmology. Amsterdam (Netherlands): Academic Press

Doran M, Robbers G. 2006. JCAP 0606:026

Drell PS, Loredo TJ, Wasserman I. 2000. Astrophys. J. 530:593-617

Dvali GR, Gabadadze G, Porrati M. 2000. Phys. Lett. B485:208-214

Efstathiou G, Sutherland WJ, Maddox SJ. 1990. Nature 348:705-707

Einstein A. 1917. Sitzungsber. K. Akad. 6:142-152

Eisenstein DJ, et al. 2005. Astrophys. J. 633:560-574

Enqvist K. 2007. astro-ph/0709.2044

Fosalba P, Gaztanaga E. 2004. Mon. Not. Roy. Astron. Soc. 350:L37-L41

Freedman WL, Madore BF, Gibson BK, Ferrarese L, Kelson DD, et al. 2001. Astrophys. J. 553:47-72

Friedman AS, Bloom JS. 2005. Astrophys. J. 627:1-25

Frieman JA, Hill CT, Stebbins A, Waga I. 1995. Phys. Rev. Lett. 75:2077-2080

Frieman JA, Huterer D, Linder EV, Turner MS. 2003. Phys. Rev. D 67:083505

Fukugita M, Futamase T, Kasai M, Turner EL. 1992. Astrophys. J. 393:3-21

Gerke BF, Efstathiou G. 2002. Mon. Not. Roy. Astron. Soc. 335:33

Gradwohl BA, Frieman JA. 1992. Astrophys. J. 398:407-424

Gregory R, Kaloper N, Myers RC, Padilla A. 2007. JHEP 10:069

Guerra EJ, Daly RA, Wan L. 2000. Astrophys. J. 544:659-670

Gunn JE, Tinsley BM. 1975. Nature 257:454-457 
Guth AH. 1981. Phys. Rev. D23:347-356

Guzik J, Bernstein G, Smith RE. 2007. MNRAS 375:1329-1337

Haiman Z, Mohr JJ, Holder GP. 2001. Astrophys. J. 553:545-561

Hamuy M, Phillips MM, Suntzeff NB, Schommer RA, Maza J, et al. 1996. Astron. J. 112:2408

Hennawi JF, Spergel DN. 2005. Astrophys. J. 624:59-79

Heymans C, White M, Heavens A, Vale C, van Waerbeke L. 2006. MNRAS 371:750-760

Hillebrandt W, Niemeyer JC. 2000. Ann. Rev. Astron. Astrophys. 38:191-230

Hirata CM, Seljak U. 2004. Phys. Rev. D 70:063526

Hoeflich P. 2004. astro-ph/0409170

Hoekstra H, et al. 2006. Astrophys. J. 647:116-127

Hoyle F. 1948. MNRAS 108:372

Hu W. 2002. Phys. Rev. D 65:023003

Hu W. 2005. ASP Conf. Ser. 339:215

$\mathrm{Hu}$ W, Dodelson S. 2002. Ann. Rev. Astron. Astrophys. 40:171-216

Hu W, Haiman Z. 2003. Phys. Rev. D68:063004

$\mathrm{Hu}$ W, Holz DE, Vale C. 2007. astro-ph/0708.4391

Hu W, Jain B. 2004. Phys. Rev. D 70:043009

Hu W, Scranton R. 2004. Phys. Rev. D70:123002

Hubble E. 1929. Proceedings of the National Academy of Science 15:168-173

Hui L, Greene PB. 2006. Phys. Rev. 73:123526

Hui L, Stebbins A, Burles S. 1999. Astrophys. J. Letters 511:L5-L8

Huterer D. 2002. Phys. Rev. D65:063001

Huterer D, Cooray A. 2005. Phys. Rev. D71:023506

Huterer D, Starkman G. 2003. Phys. Rev. Lett. 90:031301

Huterer D, Takada M, Bernstein G, Jain B. 2006. MNRAS 366:101-114

Huterer D, Turner MS. 1999. Phys. Rev. D60:081301

Huterer D, Turner MS. 2001. Phys. Rev. D64:123527

Jaffe AH, Ade PA, Balbi A, Bock JJ, Bond JR, et al. 2001. Physical Review Letters 86:3475-3479

Jain B, Taylor A. 2003. Phys. Rev. Lett. 91:141302

Jarvis M, Jain B, Bernstein G, Dolney D. 2006. Astrophys. J. 644:71-79

Jha S, Riess AG, Kirshner RP. 2007. Astrophys. J. 659:122-148

Jimenez R, Loeb A. 2002. Astrophys. J. 573:37-42

Johnston DE, Sheldon ES, Wechsler RH, Rozo E, Koester BP, et al. 2007. astroph/0709.1159

Kaiser N. 1992. Astrophys. J. 388:272-286

Kaiser N, Wilson G, Luppino GA. 2000. astro-ph/0003338 
Kim A. 2004. LBNL Report LBNL-56164

Knop RA, Aldering G, Amanullah R, Astier P, Blanc G, et al. 2003. Astrophys. J. 598:102-137

Knox L, Song YS, Tyson JA. 2006. Phys. Rev. D74:023512

Kochanek CS. 1996. Astrophys. J. 466:638

Koester BP, McKay TA, Annis J, Wechsler RH, Evrard A, et al. 2007. Astrophys. J. 660:239-255

Kolb EW, Matarrese S, Riotto A. 2006. New J. Phys. 8:322

Kolb EW, Turner MS. 1990. The early universe. Reading, MA: Addison-Wesley

Kowalski M, et al. 2008. submitted

Krauss LM, Chaboyer B. 2003. Science 299:65-70

Krauss LM, Scherrer RJ. 2007. General Relativity and Gravitation 39:1545-1550

Krauss LM, Turner MS. 1995. Gen. Rel. Grav. 27:1137-1144

Leibundgut B. 2001. Ann. Rev. Astron. Astrophys. 39:67-98

Lima M, Hu W. 2004. Phys. Rev. D 70:043504

Linder EV. 2003. Phys. Rev. Lett. 90:091301

Linder EV. 2007. astro-ph/0704.2064

Lue A, Scoccimarro R, Starkman G. 2004. Phys. Rev. D 69:044005

Ma Z, Hu W, Huterer D. 2006. Astrophys. J. 636:21-29

Majumdar S, Mohr JJ. 2004. Astrophys. J. 613:41-50

Massey R, et al. 2007. Nature 445:286

Meneghetti M, Jain B, Bartelmann M, Dolag K. 2005. Mon. Not. Roy. Astron. Soc. $362: 1301-1310$

Miknaitis G, et al. 2007. Astrophys. J. 666:674-693

Mitchell JL, Keeton CR, Frieman JA, Sheth RK. 2005. Astrophys. J. 622:81-98

Mohr JJ. 2005. In Observing Dark Energy, eds. SC Wolff, TR Lauer, vol. 339 of Astronomical Society of the Pacific Conference Series

Motl PM, Hallman EJ, Burns JO, Norman ML. 2005. Astrophys. J. Letters 623:L63-L66

Munshi D, Valageas P, Van Waerbeke L, Heavens A. 2006. astro-ph/0612667

Nagai D. 2006. Astrophys. J. 650:538-549

Ostriker JP, Steinhardt PJ. 1995. Nature 377:600-602

Padmanabhan T. 2003. Phys. Rept. 380:235-320

Peacock JA. 1999. Cosmological Physics. Cambridge, UK: Cambridge University Press

Peebles PJE. 1984. Astrophys. J. 284:439-444

Peebles PJE. 1993. Principles of physical cosmology. Princeton, NJ: Princeton University Press

Peebles PJE, Ratra B. 2003. Rev. Mod. Phys. 75:559-606

Perlmutter S, Aldering G, Goldhaber G, Knop RA, Nugent P, et al. 1999. Astrophys. J. 517:565-586 
Perlmutter S, Gabi S, Goldhaber G, Goobar A, Groom DE, et al. 1997. Astrophys. J. 483:565

Perlmutter S, Schmidt BP. 2003. In Supernovae and Gamma-Ray Bursters, ed. K Weiler, vol. 598 of Lecture Notes in Physics, Berlin Springer Verlag

Petrosian V, Salpeter E, Szekeres P. 1967. Astrophys. J. 147:1222-1226

Phillips MM. 1993. Astrophys. J. Letters 413:L105-L108

Plewa T, Calder AC, Lamb DQ. 2004. Astrophys. J. Letters 612:L37-L40

Pryke C, Halverson NW, Leitch EM, Kovac J, Carlstrom JE, et al. 2002. Astrophys. J. 568:46-51

Rapetti D, Allen SW. 2007. astro-ph/0710.0440

Rapetti D, Allen SW, Amin MA, Blandford RD. 2007. Mon. Not. Roy. Astron. Soc. 375:1510-1520

Ratra B, Peebles PJE. 1988. Phys. Rev. D37:3406

Reichardt CL, Ade PAR, Bock JJ, Bond JR, Brevik JA, et al. 2008. astroph/0801.1491 801

Riess AG. 2000. Pub. Astron. Soc. Pacific 112:1284

Riess AG, Filippenko AV, Challis P, Clocchiatti A, Diercks A, et al. 1998. Astron. J. 116:1009-1038

Riess AG, Nugent PE, Gilliland RL, Schmidt BP, Tonry J, et al. 2001. Astrophys. J. 560:49-71

Riess AG, Strolger LG, Casertano S, Ferguson HC, Mobasher B, et al. 2007. Astrophys. J. 659:98-121

Riess AG, et al. 2004. Astrophys. J. 607:665-687

Sahni V, Starobinsky A. 2006. astro-ph/0610026

Sandage A. 1962. Astrophys. J. 136:319-333

Sandage AR. 1970. Physics Today 23:34-41

Schaefer BE. 2003. Astrophys. J. Letters 583:L67-L70

Schneider P. 2006. In Saas-Fee Advanced Course 33: Gravitational Lensing: Strong, Weak and Micro, eds. G Meylan, P Jetzer, P North, P Schneider, CS Kochanek, J Wambsganss

Scranton R, Connolly AJ, Nichol RC, Stebbins A, Szapudi I, et al. 2003. astroph/0307335

Seo HJ, Eisenstein DJ. 2003. Astrophys. J. 598:720-740

Seo HJ, Eisenstein DJ. 2007. Astrophys. J. 665:14-24

Shapiro C, Turner MS. 2006. Astrophys. J. 649:563-569

Smith RE, Scoccimarro R, Sheth RK. 2007. astro-ph/0703620

Song YS, Hu W, Sawicki I. 2007. Phys. Rev. D75:044004

Spergel DN, Bean R, Doré O, Nolta MR, Bennett CL, et al. 2007. Astrophys. J. Suppl. 170:377-408

Springel V, Frenk CS, White SDM. 2006. Nature 440:1137-1144

Stanek R, Evrard AE, Bohringer HB, Schuecker P, Nord B. 2006. Astrophys. J. 648:956-968 
Starobinsky AA. 1998. JETP Lett. 68:757-763

Straumann N. 2002. gr-qc/0208027

Sunyaev RA, Zeldovich YB. 1970. Comments on Astrophysics and Space Physics 2:66-73

Susskind L. 2003. hep-th/0302219

Takada M, Jain B. 2004. Mon. Not. Roy. Astron. Soc. 348:897

Tegmark M, Eisenstein DJ, Strauss MA, Weinberg DH, Blanton MR, et al. 2006. Phys. Rev. D 74:123507

Tinsley BM, Gunn JE. 1976. Astrophys. J. 203:52-62

Tomita K. 2001. Mon. Not. Roy. Astron. Soc. 326:287

Turner MS. 1991. In Primordial Nucleosynthesis and Evolution of Early Universe, eds. K Sato, J Audouze, vol. 169 of Astrophysics and Space Science Library

Turner MS, Steigman G, Krauss LM. 1984. Phys. Rev. Lett. 52:2090-2093

Turner MS, White MJ. 1997. Phys. Rev. D56:4439-4443

Turok N, ed. 1997. Critical dialogues in cosmology. Singapore: World Scientific

Uzan JP. 2007. Gen. Rel. Grav. 39:307-342

Van Waerbeke L, Mellier Y, Erben T, Cuillandre JC, Bernardeau F, et al. 2000. Astron. 85 Astrophys. 358:30-44

Visser M. 2004. Class. Quant. Grav. 21:2603-2616

Wang L, Steinhardt PJ. 1998. Astrophys. J. 508:483-490

Wang S, Hui L, May M, Haiman Z. 2007. Phys. Rev. D76:063503

Wang Y, Mukherjee P. 2004. Astrophys. J. 606:654-663

Warren MS, Abazajian K, Holz DE, Teodoro L. 2006. Astrophys. J. 646:881-885

Weinberg S. 1987. Phys. Rev. Lett. 59:2607

Weinberg S. 1989. Rev. Mod. Phys. 61:1-23

Weller J, Albrecht A. 2002. Phys. Rev. D65:103512

Wetterich C. 1988. Nucl. Phys. B302:668

White M, van Waerbeke L, Mackey J. 2002. Astrophys. J. 575:640-649

Wittman DM, Tyson JA, Kirkman D, Dell'Antonio I, Bernstein G. 2000. Nature 405:143-148

Wood-Vasey WM, Miknaitis G, Stubbs CW, Jha S, Riess AG, et al. 2007. Astrophys. J. 666:694-715

Yee HKC, Gladders MD. 2002. In AMiBA 2001: High-Z Clusters, Missing Baryons, and CMB Polarization, eds. LW Chen, CP Ma, KW Ng, UL Pen, vol. 257 of Astronomical Society of the Pacific Conference Series

Zel'dovich YB. 1968. Sov. Phys. Usp. 11:381-393

Zentner AR, Rudd DH, Hu W. 2007. astro-ph/0709.4029

Zlatev I, Wang LM, Steinhardt PJ. 1999. Phys. Rev. Lett. 82:896-899 